\title{
Landau Hamiltonians With Random Potentials: Localization and the Density of States
}

\author{
J.M. Combes ${ }^{1,2}$, P.D. Hislop ${ }^{3,4}$ \\ Centre de Physique Théorique ${ }^{5}$, CNRS, Luminy, Case 907, F-13288 Marseille Cedex 9, France
}

Received: 11 October 1994 / Accepted: 17 July 1995

\begin{abstract}
We prove the existence of localized states at the edges of the bands for the two-dimensional Landau Hamiltonian with a random potential, of arbitrary disorder, provided that the magnetic field is sufficiently large. The corresponding eigenfunctions decay exponentially with the magnetic field and distance. We also prove that the integrated density of states is Lipschitz continuous away from the Landau energies. The proof relies on a Wegner estimate for the finite-area magnetic Hamiltonians with random potentials and exponential decay estimates for the finitearea Green's functions. The proof of the decay estimates for the Green's functions uses fundamental results from two-dimensional bond percolation theory.
\end{abstract}

\section{Introduction}

The existence of localized states for a two-dimensional gas of non-interacting electrons in a constant magnetic field is a main ingredient in various discussions and proofs of the integer quantum Hall effect (see e.g. [1-4,7]). It is generally believed that localization occurs near the band edges for large magnetic fields and bounded, random potentials of arbitrary disorder. According to Halperin's argument [1], the localization length should diverge near the Landau levels. This is in contrast to the situation with no magnetic field. For two dimensional random systems, localization is expected to hold at all energies for arbitrary disorder and the eigenfunctions are expected to decay exponentially.

In this paper, we study the family $H_{\omega}$ of two-dimensional Landau Hamiltonians with Anderson-type potentials, having mean zero, on $L^{2}\left(\mathbb{R}^{2}\right)$. We prove that localization does occur in all energy intervals $I_{n}(B) \equiv\left[(2 n+1) B+\mathcal{O}\left(B^{-1}\right)\right.$, $\left.(2 n+3) B-\mathcal{O}\left(B^{-1}\right)\right], \quad n=0,1,2, \ldots$ at large magnetic field strengths $B$ and for arbitrary disorder. Recall that $\sigma\left(H_{\omega}\right)$ is contained in bands about the Landau levels

\footnotetext{
${ }^{1}$ Département de Mathématiques, Université de Toulon et du Var, F-83130 La Garde, France.

2 Supported in part by CNRS.

3 Mathematics Department, University of Kentucky, Lexington KY 40506-0027, USA.

4 Supported in part by NSF grants INT 90-15895 and DMS 93-07438.

${ }^{5}$ Unité Propre de Recherche 7061.
} 
$E_{n}(B) \equiv(2 n+1) B, n=0,1,2, \ldots$, of width $\|V\|_{\infty}$, independent of $B$. We follow the approach of [8] developed to study random Schrödinger operators on $L^{2}\left(\mathbb{R}^{d}\right)$. This work [8] extends to continuous systems the techniques of Howland [9], SimonWolff [10], von Dreifus-Klein [11], and Spencer [12].

For large magnetic fields, we justify the one Landau band approximation of [13] for each Landau band and obtain exponential decay estimates in $x$ and $B$ on the Green's function for finite-area Hamiltonians. The key to these estimates is showing that equipotential lines, defined by $E=V(x)+E_{n}(B)$ for energies $E$ in the band edges, don't percolate with high probability. For potentials with zero averages, this holds at all energies except the Landau levels, which correspond to the critical percolation threshold. In addition to this restriction, there is a small region of energy of $\mathcal{O}\left(B^{-1}\right)$ around each Landau level where small denominators in the interband perturbation expansion can't be controlled by our method. Although this is in agreement with an earlier conjecture of Laughlin [14], it remains an open question whether these small bands of energies about the Landau levels correspond to extended states.

In Sect. 2 below, we describe the model and state the main results. We also give some elementary estimates needed later to justify the one Landau band approximation. In Sect. 3, we prove Wegner estimates for the quantum Hall Hamiltonian restricted to finite boxes. As a by-product, we obtain the Lipschitz continuity of the integrated density of states away from the Landau energies. The proofs of the exponential decay of the finite-area Green's function are given in Sect. 4. The results of Sect. 3 on the Wegner estimate and Sect. 4 on the decay of the finite volume Hamiltonians are used in Sect. 5, together with results of [8], to prove the main theorem. We prove some technical lemmas in Appendix 1. In Appendix 2, we give a theorem which identifies the almost sure spectrum of the Hamiltonians $H_{\omega}$.

We have recently learned of some related results on localization for the models studied here by J. Pulé [24] and by W.M. Wang [17].

\section{The Model and the Main Results}

We consider a one-particle Hamiltonian which describes an electron in twodimensions $\left(x_{1}, x_{2}\right)$ subject to a constant magnetic field of strength $B>0$ in the perpendicular $x_{3}$-direction, and a random potential $V_{\omega}$. The Hamiltonian $H_{\omega}$ has the form

$$
H_{\omega}=(p-A)^{2}+V_{\omega},
$$

on the Hilbert space $L^{2}\left(\mathbb{R}^{2}\right)$, where $p \equiv-i \nabla$, and the vector potential $A$ is

$$
A=\frac{B}{2}\left(x_{2},-x_{1}\right) \text {, }
$$

so the magnetic field $B=\nabla \times A$ is in the $x_{3}$-direction. The random potential $V_{\omega}$ is Anderson-like having the form

$$
V_{\omega}(x)=\sum_{i \in \mathbb{Z}^{2}} \lambda_{i}(\omega) u(x-i) .
$$

We make the following assumptions on the single-site potential $u$ and the coupling constants $\left\{\lambda_{i}(\omega)\right\}$. 
(V1) $u \geqq 0, \quad u \in C^{2}, \quad \operatorname{supp} u \subset B\left(0, \frac{1}{\sqrt{2}}\right), \quad$ and $\quad \exists C_{0}>0 \quad$ and $\quad r_{0}>0 \quad$ s.t. $u \mid B\left(0, r_{0}\right)>C_{0}$.

(V2) $\left\{\lambda_{i}(\omega)\right\}$ is an independent, identically distributed family of random variables with common distribution $g \in C^{2}([-M, M])$, for some $0<M<\infty$, s.t. $g(-\lambda)=g(\lambda)$ and $g(\lambda)>0$ Lebesgue a.e. $\lambda \in[-M, M]$.

Note that these conditions imply that $\left\|V_{\omega}\right\|_{\infty} \leqq M_{0} \equiv 2\|u\|_{\infty} M$. We denote by $H_{A} \equiv(p-A)^{2}$, the Landau Hamiltonian. As is well-known, the spectrum of $H_{A}$ consists of an increasing sequence $\left\{E_{n}(B)\right\}$ of eigenvalues, each of infinite multiplicity, given by

$$
E_{n}(B)=(2 n+1) B, \quad n=0,1,2, \ldots .
$$

Note that $D\left(H_{\omega}\right)=D\left(H_{A}\right) \forall \omega \in \Omega$, the probability space for the model. We will call $E_{n}(B)$ the $n^{\text {th }}$ Landau level and denote by $P_{n}$ the projection onto the corresponding subspace. The orthogonal projection is denoted by $Q_{n} \equiv 1-P_{n}$. Let $M_{0} \equiv$ $\sup _{x, \omega}\left|V_{\omega}(x)\right|<\infty$. Then, $\sigma\left(H_{\omega}\right) \subset \bigcup_{n \geqq 0} \sigma_{n}$, where $\sigma_{n} \equiv\left[E_{n}(B)-M_{0}, E_{n}(B)+M_{0}\right]$, which we call the $n^{\text {th }}$ Landau band. We show that $\sigma\left(H_{\omega}\right)$ is deterministic. The magnetic translations are defined for $a \in \mathbb{Z}^{2}$ by

$$
U_{a} \equiv e^{-i B x \wedge a} e^{-i p \cdot a},
$$

where $x \wedge a \equiv x_{2} a_{1}-x_{1} a_{2}$. We then have

$$
U_{a} H_{\omega} U_{a}^{-1}=H_{T_{a} \omega},
$$

where $T_{a}: \Omega \rightarrow \Omega$ is the $\mathbb{Z}^{2}$-translation. Standard results (cf. [15]) show that $H_{\omega}$ is a $\mathbb{Z}^{2}$-ergodic self-adjoint family of operators and consequently its spectrum is deterministic. Note that $\sigma\left(H_{\omega}\right)$ is not necessarily equal a.s. to $\bigcup_{n \geqq 0} \sigma_{n}$. In Appendix 2, we show that the family $H_{\omega}$ almost surely has spectrum away from the Landau levels in the following sense. For any $N>0$, there exists constants $B_{N}>0$ and $C_{N}>0$, such that for all $B>B_{N}>0$, the complement of the set $\bigcup_{n \leqq N}\left(E_{n}(B)-C_{N} B^{-1 / 2}, E_{n}(B)+C_{N} B^{-1 / 2}\right)$ in $\left[0, E_{N}(B)+M_{0}+C_{N} B^{-1 / 2}\right]$ contains spectrum with probability one.

Theorem 2.1. Let $H_{\omega}$ be the family given in (2.1) with vector potential A satisfying (2.2), $B>0$, and the random potential $V_{\omega}$ as in (2.3) and satisfying (V1)-(V2). Let $I_{n}(B)$ denote the interval

$$
I_{n}(B) \equiv\left[E_{n}(B)+\mathcal{O}\left(B^{-1}\right), E_{n+1}(B)-\mathcal{O}\left(B^{-1}\right)\right],
$$

where the term $\mathcal{O}\left(B^{-1}\right)$ depends on $n$. There exists $B_{0} \gg 0$ such that for $B>B_{0}$ and all $n=-1,0,1,2, \ldots$,

$$
\sigma\left(H_{\omega}\right) \cap I_{n}(B)
$$

is pure point almost surely and the corresponding eigenfunctions decay exponentially. The integrated density of states is Lipschitz continuous away from $\sigma\left(H_{A}\right)$.

Let us make two remarks about the theorem. First, we note that the above theorem holds at arbitrary disorder. For large disorder, the techniques of [8] apply directly to show that, without the percolation estimates, $\sigma\left(H_{\omega}\right)$ is almost surely pure point in each Landau band. This regime, however, is of little interest as the quantum Hall conductivity vanishes in this case. Secondly, we show, in fact, that the localization length, for energies near the band edges as in Theorem 2.1, is a decreasing 
function of the field strength $B$ so that the wave functions are strongly localized. We also show that the localization length increases as the energy approaches the Landau levels. The precise manner in which this occurs follows from Proposition 5.1 and Theorem 5.2. However, our method fails to give an estimate of the power law divergence of the localization length near the Landau level.

As is clear from the Wegner estimate, Theorem 3.1, our method fails to give information about the integrated density of states at the Landau energies. However, we can improve the result if, instead of the support condition of (V1), we assume that the function $u$ is strictly positive on the unit square $\Lambda_{1}(0)$ centered at the origin. In general, we use the notation $\Lambda_{l}(x)$ for the square of side $l$ centered at $x$ and $\chi_{\Lambda_{l}(x)}$ for the characteristic function for this square.

Theorem 2.2. Let $H_{\omega}$ be the family given in (2.1) with vector potential A satisfying (2.2), $B>0$, and the random potential $V_{\omega}$ as in (2.3). Assume condition (V1) and (V3) $u \geqq 0, u \in C_{0}^{2}$, and $\exists C_{0}>0$ such that $u \mid B(0,1)>C_{0}$.

\section{Then, the integrated density of states is Lipschitz continuous.}

If the hypothesis of Theorem 2.2 does not hold, then a large portion of configuration space is unaffected by the potential. It is not, therefore, surprising that there is a discontinuity in the integrated density of states at the Landau energies as there is for the Landau Hamiltonian. A phenomenon of this type has been observed by Brézin et al. [6] for a Poisson distribution of impurities at low energy. So we do not expect that the IDS is Lipschitz continuous at the Landau energies without a condition of the support of $u$ which implies that the zero set of $V_{\omega}$ is in some sense "small."

We mention that W.M. Wang [16] has obtained an asymptotic expansion in the semi-classical limit for the density of states at large magnetic field strengths away from the Landau levels, partially justifying the one-band approximation.

We conclude this section with some simple observations on the Landau projections $P_{n}$.

The projection $P_{n}$ on the $n^{\text {th }}$ Landau level of $H_{A}$ has a kernel given by

$$
P_{n}(x, y)=B e^{-\frac{i B}{2} x \wedge y} p_{n}\left(B^{\frac{1}{2}}(x-y)\right),
$$

where $p_{n}(x)$ is of the form

$$
p_{n}(x)=\left\{n^{\text {th }} \text { degree polynomial in } x\right\} e^{-\frac{|x|^{2}}{2}},
$$

and independent of $B$. We will make repeated use of the following elementary lemma, the proof of which follows by direct calculation using the kernel (2.7)-(2.8).

Lemma 2.1. Let $\chi_{1}, \chi_{2}$ be functions of disjoint, not necessarily compact, support with $\left|\chi_{i}\right| \leqq 1$, and let $\delta \equiv \operatorname{dist}\left(\operatorname{supp} \chi_{1}, \operatorname{supp} \chi_{2}\right)>0$. Then,

(1) $\left\|\chi_{1} P_{n} \chi_{1}\right\|_{1} \leqq C_{n} B\left|\operatorname{supp} \chi_{1}\right|$;

(2) $\left\|\chi_{1} P_{n} \chi_{2}\right\|_{H S} \leqq C_{n} B^{1 / 2} e^{-B \delta^{2} / 8} \inf \left\{\left|\operatorname{supp} \chi_{1}\right|,\left|\operatorname{supp} \chi_{2}\right|\right\}^{\frac{1}{2}}$,

where $C_{n}$ varies from line to line and depends only on $n$, and HS denotes the Hilbert Schmidt norm. 


\section{Wegner Estimate}

We define local Hamiltonians as relatively compact perturbations of the Landau Hamiltonian $H_{A}=(p-A)^{2}$, as defined in Sect. 2. Let $\Lambda \subset \mathbb{R}^{2}$ denote an open connected region in $\mathbb{R}^{2}$. We let $\Lambda_{l}(x)$ denote a square of side $l$ centered at $x \in \mathbb{R}^{2}$,

$$
\Lambda_{l}(x) \equiv\left\{y \in \mathbb{R}^{2}|| x_{i}-y_{i} \mid<l, i=1,2\right\}
$$

Given $\Lambda \subset \mathbb{R}^{2}$, the local potential $V_{\Lambda}$ is defined as follows. Freeze all $\lambda_{j}(\omega) \in$ $\mathbb{Z}^{2} \cap\left(\mathbb{R}^{2} \backslash \Lambda\right)$ and consider $\tilde{V}$ so obtained. This potential depends on the external, fixed coupling constants and on all $\lambda_{i}(\omega) \in \mathbb{Z}^{2} \cap \Lambda$. We define $V_{\Lambda} \equiv \tilde{V} \mid \Lambda$ and define $H_{\Lambda} \equiv H_{A}+V_{\Lambda}$ on $L^{2}\left(\mathbb{R}^{2}\right)$. We denote by $\mathbb{P}_{\Lambda}$ and $\mathbb{E}_{\Lambda}$ the probability and expectation with respect to the random variables in $\Lambda$. Note that $\sigma_{\text {ess }}\left(H_{\Lambda}\right)=\sigma_{\text {ess }}\left(H_{A}\right)$, since $V_{A}$ is relatively compact.

For general regions $\Lambda$, the Hamiltonians $H_{\Lambda}$ are not independent of the external configurations. This lack of independence is characteristic of models on $\mathbb{R}^{d}$. The multi-scale analysis of [8] requires that we compute the simultaneous occurrence of events localized in several disjoint regions. For example, we must estimate $\mathbb{P}_{\Lambda}(A \cap B)$ in terms of $\mathbb{P}_{\Lambda}(A)$ and $\mathbb{P}_{\Lambda}(B)$. This is particularly difficult when the singlesite potential $u$ does not have compact support. For a discussion of these issues, we refer the reader to [20] (the argument of [8] for the case when $u$ has non-compact support is incomplete; the complete argument can be found in [20]). The results of [20] are not needed in the present situation (or, when the $\operatorname{supp} u$ is compact, in general). Condition (V1) states that $\operatorname{supp} u \subset B\left(0, \frac{1}{\sqrt{2}}\right)$. In the multi-scale analysis of Sect. 5, the regions $\Lambda$ are chosen to be squares in the lattice $\Gamma \equiv e^{i \pi / 4} \sqrt{2} \mathbb{Z}^{2}$. This lattice is chosen in order to apply results of bond percolation theory, see Sect. 4. Each lattice site of $\mathbb{Z}^{2}$ is a center of a bond in the lattice $\Gamma$ (see Fig. 1). Let $\Lambda$ be a square region with edges parallel to the bonds of $\Gamma$ and corners at vertices of $\Gamma$. Let $\Lambda$ be the subregion obtained by deleting the edge region of $\Lambda$, that is $\hat{\Lambda} \equiv \Lambda /\{x \in \Lambda \mid\|x-y\|<1 / \sqrt{2} \forall y \in \partial \Lambda\}$. We define a local potential associated with $\Lambda$ to be $V_{\Lambda} \equiv \sum_{i \in \hat{\Lambda} \cap \Gamma} \lambda_{i}(\omega) u(x-i)$. Since the support of the single-site potential $u$ is contained entirely within the basic cell for this lattice $\Gamma$, it is clear that the support of $\hat{V}_{\Lambda}$ is contained entirely within $\Lambda$. Furthermore, the potentials $\hat{V}_{\Lambda_{1}}$ and $\hat{V}_{\Lambda_{2}}$, associated with disjoint square regions $\Lambda_{1}$ and $\Lambda_{2}$ with vertices lying in $\Gamma$, are independent. Consequently, for these square regions, if $A$ and $B$ represent

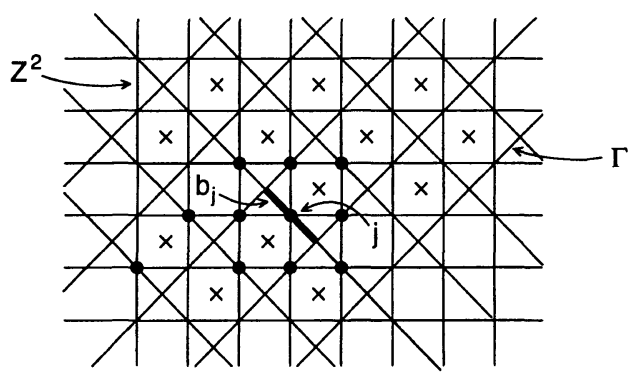

Fig. 1. The lattice $\Gamma$ and the bond $b_{j}, j \in \mathbb{Z}^{2}$. 
events associated with the potentials $\hat{V}_{\Lambda_{1}}$ and $\hat{V}_{\Lambda_{2}}$, we have the usual equality,

$$
\mathbb{P}_{\hat{\Lambda}}(A \cap B)=\mathbb{P}_{\hat{\Lambda}}(A) \mathbb{P}_{\hat{\Lambda}}(B),
$$

and its extension to multiple events. We define local Hamiltonians $\hat{H}_{\Lambda} \equiv H_{\Lambda}+\hat{V}_{\Lambda}$ associated with these potentials. We will use these local Hamiltonians in the multiscale analysis. Note that if $\Lambda_{1} \subset \Lambda_{2}$, then $\hat{V}_{\Lambda_{2}} \mid \Lambda_{1}-\hat{V}_{\Lambda_{1}}$ is supported near the boundary of $\Lambda_{1}$ and its bound is independent of the regions. Consequently, we can absorb this term into the operator $W(\chi)$, appearing in the geometric resolvent equation (see Sect. 5), with the only effect of changing the constants. Theorem 3.1 and the results of Sect. 4 hold as well for these local Hamiltonians $\hat{H}_{\Lambda}$.

We prove the following theorem.

Theorem 3.1. $\exists B_{0}>0$ and a constant $C_{W}>0$ such that for all $B>B_{0}$ and for any $E \notin \sigma\left(H_{A}\right)$,

$$
\mathbb{P}_{\Lambda}\left\{\operatorname{dist}\left(\sigma\left(H_{\Lambda}\right), E\right)<\delta\right\} \leqq C_{W}\left[\operatorname{dist}\left(\sigma\left(H_{A}\right), E\right)-\delta\right]^{-2}\|g\|_{\infty} \delta B|\Lambda| .
$$

This theorem will follow from the properties of the spectral projectors for $H_{A}$ and a spectral averaging theorem. Since $H_{\Lambda}$ depends analytically on the coupling constants $\lambda_{i}$, we need only a simple version of the spectral averaging theorem which we state without proof (cf. $[8,18,19])$.

Lemma 3.1. Let $U$ be a bounded, self-adjoint operator for which there exist a constant $C_{0}>0$ and a bounded, self-adjoint operator $D$ such that $C_{0} D^{2} \leqq U<\infty$. We define a self-adjoint family $H_{\lambda}, \lambda \in \mathbb{R}$, on $D\left(H_{0}\right)$ by $H_{\lambda} \equiv H_{0}+\lambda U$. Let $E_{\lambda}(\cdot)$ be the spectral family for $H_{\lambda}$. For any $h \geqq 0$, supp $h$ compact, and $h \in L^{\infty}(\mathbb{R})$, and for any $L \subset \mathbb{R}$ measurable, we have

$$
\left\|\int_{\mathbb{R}} h(\lambda) D E_{\lambda}(L) D d \lambda\right\| \leqq C_{0}^{-1}|L|\|h\|_{\infty} .
$$

In Sect. 5, we will apply this lemma to the family $H_{\lambda}=H_{0}+\lambda u$, where $u$ is a single-site potential satisfying conditions (V1), and $\lambda=\lambda_{0}(\omega)$ is distributed as in condition (V2). In this case, we may take $D$ to be the characteristic function for the disk $B\left(0, r_{0}\right)$.

For simplicity, we will work with the case $n=0$, the first Landau band, although the calculation is the same for $n$, with constants depending on $n$. As can be easily checked, the calculations depend only on the difference between the energy $E$ that we are considering and the nearest Landau energy $E_{n}(B)$. To begin the proof of Theorem 3.1, we need a simple estimate. Let $\Delta$ be an interval in the first Landau band $\sigma_{0}$. Let $E_{\Delta}$ be the spectral projector for $H_{\Lambda}$ associated with $\Delta$ and let $Q_{0} \equiv 1-P_{0}$, as defined in Sect. 2 .

Lemma 3.2. $\left\|E_{\Delta} Q_{0} E_{\Delta}\right\| \leqq d_{\Delta}^{-2}\left(1-\left(2 d_{\Delta}\right)^{-1}|\Delta|\right)^{-2} M_{0}^{2}$, where $d_{\Delta} \equiv \operatorname{dist}\left(\sigma\left(H_{A}\right) \backslash\{B\}\right.$, $\Delta)=\mathcal{O}(B)$, and $M_{0} \equiv\left\|\bar{V}_{\omega}\right\|_{\infty} \leqq M\|u\|_{\infty}$.

Proof. Let $E_{m} \in \Delta$ be the center of the interval. We then can write

$$
\begin{aligned}
E_{\Delta} Q_{0} E_{\Delta} & \leqq\left[\operatorname{dist}\left(\sigma\left(H_{A}\right) \backslash\{B\}, \Delta\right)\right]^{-1}\left(E_{\Delta}\left(H_{A}-E_{m}\right) Q_{0} E_{\Delta}\right) \\
& \leqq d_{\Delta}^{-1}\left\{E_{\Delta}\left(H_{\Lambda}-E_{m}\right) Q_{0} E_{\Delta}+E_{\Delta} V_{\Lambda} Q_{0} E_{\Delta}\right\}
\end{aligned}
$$


This implies that

$$
\left\|E_{\Delta} Q_{0} E_{\Delta}\right\| \leqq d_{\Delta}^{-1}\left\{\frac{|\Delta|}{2}\left\|E_{\Delta} Q_{0} E_{\Delta}\right\|+M_{0}\left\|Q_{0} E_{\Delta}\right\|\right\} .
$$

Since $d_{\Delta}=\mathcal{O}(B)$, it is clear that for all $B$ sufficiently large $\left(2 d_{\Delta}\right)^{-1}|\Delta| \ll 1$, so

$$
\left\|E_{\Delta} Q_{0} E_{\Delta}\right\| \leqq d_{\Delta}^{-1}\left(1-\left(2 d_{\Delta}\right)^{-1}|\Delta|\right)^{-1} M_{0}\left\|E_{\Delta} Q_{0} E_{\Delta}\right\|^{\frac{1}{2}},
$$

and the result follows.

Note that as $d_{\Delta}=\mathcal{O}(B)$, we obtain

$$
\left\|E_{\Delta} Q_{0} E_{\Delta}\right\|=\mathcal{O}\left(B^{-2}\right) .
$$

Proof of Theorem 3.1. We can assume without loss of generality that the closest point in $\sigma\left(H_{A}\right)$ to $E$ is $E_{0}(B)=B$. All the calculations below hold for any band. Let $\Delta \subset \sigma_{0} \backslash\left\{E_{0}(B)\right\}$ be a connected interval containing $E$ and let $E_{\Delta}$ be the spectral projection for $H_{\Lambda}$ and $\Delta$. Recall from Chebyshev's inequality that

$$
\mathbb{P}_{\Lambda}\left\{\operatorname{dist}\left(\sigma\left(H_{\Lambda}\right), E\right)<\delta\right\} \leqq \mathbb{E}_{\Lambda}\left(\operatorname{Tr} E_{\Delta}\right),
$$

where $\mathbb{P}_{\Lambda}$ and $\mathbb{E}_{\Lambda}$ denote the probability and expectation with respect to the variables in $\Lambda \cap \mathbb{Z}^{2}, \operatorname{Tr}$ denotes the trace on $L^{2}\left(\mathbb{R}^{2}\right)$, and $\delta$ is such that $[E-\delta, E+\delta] \subset \Delta$. We first note that

$$
\operatorname{Tr} E_{\Delta} \leqq 2 \operatorname{Tr}\left(P_{0} E_{\Delta} P_{0}\right) .
$$

This follows from the identity

$$
\operatorname{Tr} E_{\Delta}=\operatorname{Tr} E_{\Delta} P_{0} E_{\Delta}+\operatorname{Tr} E_{\Delta} Q_{0} E_{\Delta}
$$

and the bound

$$
\operatorname{Tr} E_{\Delta} Q_{0} E_{\Delta} \leqq\left\|E_{\Delta} Q_{0} E_{\Delta}\right\|\left(\operatorname{Tr} E_{\Delta}\right),
$$

since $E_{\Delta} Q_{0} E_{\Delta} \geqq 0$. Now by Lemma 3.2, $\left\|E_{\Delta} Q_{0} E_{\Delta}\right\|=\mathcal{O}\left(B^{-2}\right)$, so (3.4) follows for all $B$ sufficiently large. Let us now suppose inf $\Delta>B$ for definiteness. From (3.4), and positivity we obtain

$$
\begin{aligned}
\operatorname{Tr} E_{\Delta} P_{0} E_{\Delta} & \leqq \operatorname{Tr}\left(E_{\Delta}\left(H_{\Lambda}-B\right) P_{0}\left(H_{\Lambda}-B\right) E_{\Delta}\right) \cdot[\operatorname{dist}(\Delta, B)]^{-2} \\
& \leqq \operatorname{Tr}\left(P_{0} V_{\Lambda} E_{\Delta} V_{\Lambda} P_{0}\right) \cdot[\operatorname{dist}(\Delta, B)]^{-2}
\end{aligned}
$$

Writing $V_{\Lambda}=\sum_{i} \lambda_{i} u_{i}$ for short, the trace in (3.5) is

$$
\sum_{i, j} \lambda_{i} \lambda_{j} \operatorname{Tr}\left(P_{0} u_{i} E_{\Delta} u_{j} P_{0}\right)
$$

where $i, j \in \Lambda \cap \mathbb{Z}^{2}$. Defining $A^{i j} \equiv u_{i}^{1 / 2} A u_{j}^{1 / 2}$ for any $A \in B(\mathscr{H})$, we have from (3.6),

$$
\sum_{i, j} \lambda_{i} \lambda_{j} \operatorname{Tr}\left(P_{0}^{j i} E_{\Delta}^{i j}\right)
$$


We must estimate

$$
\begin{aligned}
\mathbb{E}_{\Lambda}\left(\sum_{i, j} \lambda_{i} \lambda_{j} \operatorname{Tr}\left(P_{0}^{j i} E_{\Delta}^{i j}\right)\right) & \leqq \sum_{i, j} \mathbb{E}_{\Lambda}\left(\left|\lambda_{i} \lambda_{j}\right|\left|\operatorname{Tr}\left(P_{0}^{j i} E_{\Delta}^{i j}\right)\right|\right) \\
& \leqq M^{2} \sum_{i, j} \mathbb{E}_{\Lambda}\left(\left|\operatorname{Tr}\left(P_{0}^{j i} E_{\Delta}^{i j}\right)\right|\right)
\end{aligned}
$$

To estimate $\mathbb{E}_{\Lambda}\left(\left|\operatorname{Tr}\left(P_{0}^{j i} E_{\Delta}^{i j}\right)\right|\right)$, we write the spectral decomposition of the trace-class operator $P_{0}^{j i}$ as

$$
P_{0}^{j i}=\sum_{n=1}^{\infty} \mu_{n}\left\langle\psi_{n}, \cdot\right\rangle \phi_{n}
$$

where $\left\{\mu_{n}\right\}$ are the deterministic, non-negative eigenvalues of $\left|P_{0}^{j i}\right|$, so that $\sum_{n} \mu_{n}=\left\|P_{0}^{j i}\right\|_{1}$, and the set $\left\{\psi_{n}\right\}$ (respectively, $\left\{\phi_{n}\right\}$ ) is the orthonormal basis of eigenvectors for $\left|P_{0}^{j i}\right|$ (respectively, $\left|\left(P_{0}^{j i}\right)^{*}\right|=\left|P_{0}^{i j}\right|$ ). Substituting this into the trace, we obtain,

$$
\left|\operatorname{Tr}\left(P_{0}^{j i} E_{\Delta}^{i j}\right)\right| \leqq \sum_{n} \mu_{n}\left|\left\langle\psi_{n}, E_{\Delta}^{i j} \phi_{n}\right\rangle\right| \leqq \frac{1}{2} \sum_{n} \mu_{n}\left(\left\langle\phi_{n}, E_{\Delta}^{j j} \phi_{n}\right\rangle+\left\langle\psi_{n}, E_{\Delta}^{i i} \psi_{n}\right\rangle\right) .
$$

The expectation on the right side of (3.8) can be bounded above by

$$
\mathbb{E}_{\Lambda}\left(\left|\operatorname{Tr}\left(P_{0}^{j i} E_{\Delta}^{i j}\right)\right|\right) \leqq \frac{1}{2}\left\|P_{0}^{j i}\right\|_{1} \sup _{n} \mathbb{E}_{\Lambda}\left(\left\langle\phi_{n}, E_{\Delta}^{j j} \phi_{n}\right\rangle+\left\langle\psi_{n}, E_{\Delta}^{i i} \psi_{n}\right\rangle\right)
$$

We estimate the two inner products on the right side of (3.10) using Lemma 3.1 with $D$ equal to $u_{j}^{1 / 2}$ and $u_{i}^{1 / 2}$, respectively. Consequently, (3.8) is bounded above by

$$
M^{2}\|g\|_{\infty}|\Delta| \sum_{i, j}\left\|P_{0}^{j i}\right\|_{1}
$$

since $C_{0}=1$.

To evaluate the sum, we first consider those indices $i$ and $j$ for which $|i-j|<2$. Let $\chi_{i j}$ be the characteristic function for $\operatorname{supp}\left(u_{i}+u_{j}\right)$. Then the contribution from these indices to the sum in (3.9) is

$$
\sum_{|i-j|<2}\left\|P_{0}^{j i}\right\|_{1} \leqq\|u\|_{\infty}^{2} \sum_{|i-j|<2}\left\|\chi_{i j} P_{0} \chi_{i j}\right\|_{1} \leqq C_{1} B|\Lambda||\operatorname{supp} u|
$$

by Lemma 2.1, part (1). Next, in order to estimate the sum over the complimentary set of indices, we define the function $\chi_{i j}^{+}$to be the characteristic function for the set $\left\{x \in R^{2}|| x-i|<| x-j \mid\right\}$, and write $\chi_{i j}^{-} \equiv 1-\chi_{i j}^{+}$. Using the inequality

$$
\|A B\|_{1} \leqq\|A\|_{H S}\|B\|_{H S}
$$

we obtain

$$
\left\|P_{0}^{j i}\right\|_{1} \leqq\left\|u_{j}^{1 / 2} P_{0} \chi_{i j}^{+}\right\|_{H S}\left\|\chi_{i j}^{+} P_{0} u_{i}^{1 / 2}\right\|_{H S}+\left\|u_{j}^{1 / 2} P_{0} \chi_{i j}^{-}\right\|_{H S}\left\|\chi_{i j}^{-} P_{0} u_{i}^{1 / 2}\right\|_{H S} .
$$

If $|i-j| \geqq 2$, condition (V1) on the support of $u_{j}$ implies that

$$
\operatorname{dist}\left(\operatorname{supp} \chi_{i j}^{+}, \operatorname{supp} u_{j}\right) \geqq \frac{|i-j|}{2}-\frac{1}{\sqrt{2}} \geqq a|i-j|,
$$


for some strictly positive constant $a$. A similar inequality holds for dist (supp $\chi_{i j}^{-}$, $\operatorname{supp} u_{i}$ ). By part (2) of Lemma 2.1, one obtains from (3.14) and (3.15),

$$
\left\|P_{0}^{j i}\right\|_{1} \leqq 2 C_{0}^{2} B|\operatorname{supp} u| e^{-a B|t-j|^{2} / 8},
$$

from which it follows that

$$
\sum_{|i-j| \geqq 2}\left\|P_{0}^{j i}\right\|_{1} \leqq C_{2}|\operatorname{supp} u||\Lambda| .
$$

Combining (3.12) and (3.17) in (3.9), we obtain an upper bound for all $B$ large enough,

$$
\mathbb{E}_{\Lambda}\left(\operatorname{Tr} E_{\Delta}\right) \leqq C_{W}[\operatorname{dist}(B, E)-\delta]^{-2} B\|g\|_{\infty}|\Delta||\Lambda|,
$$

where $C_{W}$ depends on $M,\|u\|_{\infty}$, and supp $u$. This proves the theorem.

The estimate of Theorem 3.1 suffices to prove the Lipschitz continuity of the integrated density of states away from the Landau levels, as stated in Theorem 2.1. With regard to Theorem 2.2, let us show how the hypothesis (V3) on $\operatorname{supp} u$ allows the improvement. For $M_{0} \equiv\left\|V_{\omega}\right\|_{\infty}$ as in Sect. 2, define

$$
H_{0}=H_{A}+2 M_{0}\left(1-\chi_{A}\right) \text {, }
$$

and the finite-area Hamiltonian by

$$
H_{\Lambda}=H_{0}+V_{\Lambda} .
$$

Beginning with (3.4), we have for $\Delta \subset \sigma_{0}$ and $E_{m} \equiv$ center of $\Delta$,

$$
\begin{aligned}
\operatorname{Tr} E_{\Delta} & \leqq 2 \operatorname{Tr}\left\{E_{\Delta}\left(H_{A}+2 M_{0}-E_{m}\right) P_{0}\left(B+2 M_{0}-E_{m}\right)^{-1}\right\} \\
& \leqq 2\left(B+2 M_{0}-E_{m}\right)^{-1}\left\{\operatorname{Tr} E_{\Delta}\left(H_{\Lambda}-E_{m}\right) P_{0}+\operatorname{Tr} E_{\Delta}\left(2 M_{0} \chi_{\Lambda}-V_{\Lambda}\right) P_{0}\right\} .
\end{aligned}
$$

Since $2 M_{0} \chi_{\Lambda}-V_{\Lambda}>M_{0} \chi_{\Lambda}$ and $\left\|\left(H_{\Lambda}-E_{m}\right) E_{\Delta}\right\| \leqq \frac{|\Delta|}{2}$, we obtain

$$
\operatorname{Tr} E_{\Delta} \leqq 2\left(B+2 M_{0}-E_{m}\right)^{-1}\left\{\frac{|\Delta|}{2} \operatorname{Tr} P_{0} E_{\Delta}+M_{0} \operatorname{Tr} E_{\Delta} \chi_{\Lambda} P_{0}\right\} .
$$

As $\left(B+2 M_{0}-E_{m}\right)^{-1}|\Delta|<\frac{|\Delta|}{M_{0}} \ll 1$, we arrive at

$$
\operatorname{Tr} E_{\Delta} \leqq 4 M_{0} C_{1}^{-1}\left(B+2 M_{0}-E_{m}\right)^{-1}\left\{\sum_{i \in \Lambda \cap \mathbb{Z}^{2}} \operatorname{Tr}\left(E_{\Delta} u_{i} P_{0}\right)\right\} .
$$

Here we used the fact that $\sum_{i \in \mathbb{Z}^{2} \cap \Lambda} u_{i} \geqq C_{1} \chi_{\Lambda}$. The remaining steps are the same as above. In light of this calculation one might speculate that the singularity at the Landau energies of the IDS is due to the existence of large regions where there is no potential. Indeed, numerical studies on the Poisson model [6] seem to also support this idea.

\section{Percolation Theory and Decay Estimates}

In this section, we prove the technical estimates required to justify the oneLandau band approximation. We consider for simplicity the first Landau band 
$\sigma_{0} \equiv\left[B-M_{0}, B+M_{0}\right]$, but all other bands can be analyzed using the same techniques. Formally, if one neglects the band interaction, the effective Hamiltonian for an electron at energy $E$ is $E=B+V(x)$. Consequently, in this approximation, the electron motion is along equipotential lines $V(x)+B-E=0$. Since $V$ is random, it is natural to estimate the probability that these equipotential lines percolate through a given box. If not, the electron will remain confined to bounded regions. One can expect that the interband interaction will not change this picture. We will do this in the second part of this section by showing that the Green's function decays exponentially in $x$ and $B$ through regions where $|V(x)+B-E|>a>0$. The first part of this section is devoted to reformulating our problem as a problem in bond percolation.

4.1. Percolation Estimates. We first show that in annular regions between boxes of side $l$ and $l / 3$ there exist closed, connected ribbons where the condition $|V(x)+B-E|>a>0$ is satisfied, provided $E \neq B$, with a probability which converges exponentially fast to 1 as $l$ tends to infinity. Obviously, the existence of such a ribbon is equivalent to the impossibility for equipotential lines at energy $E$ to percolate from the center of the box to its boundary. Although this is a classical matter, let us recall how one can formulate the above condition in terms of two-dimensional bond percolation.

Recall that $V_{\omega}(x)=\sum_{i \in \mathbb{Z}^{2}} \lambda_{i}(\omega) u(x-i)$, where the single-site potential $u \geqq 0$ and has support inside a ball of radius $r_{u}<\frac{1}{\sqrt{2}}$. We define $r_{u}$ to be the smallest radius such that $\operatorname{supp} u \subset B\left(0, r_{u}\right)$. Consider a new square lattice $\Gamma \equiv e^{i \pi / 4} \sqrt{2} \mathbb{Z}^{2}$. The midpoint of each bond of $\Gamma$ is a site of $\mathbb{Z}^{2}$ (see Fig. 1). We will denote by $b_{j}$ the bond of $\Gamma$ having $j \in \mathbb{Z}^{2}$ as its midpoint. For definiteness, we assume $E \in\left(B, B+M_{0}\right)$. The other energy interval can be treated similarly.

Definition 4.1. The bond $b_{j}$ of $\Gamma$ is occupied if $\lambda_{j}(\omega)<\frac{E-B}{2}$. The probability $\mathbb{P}\left\{\lambda_{j}(\omega)<\frac{E-B}{2}\right\} \equiv p$ is the probability that $b_{j}$ is occupied ( $p$ is independent of $j$ by the iid assumption).

Let us assume that the bond $b_{j}$ is occupied and consider (see Fig. 2),

$$
\mathscr{R}_{j} \equiv\left\{x \mid \operatorname{dist}\left(x, b_{j}\right)<\frac{1}{\sqrt{2}}-r_{u} \equiv r_{1}\right\}
$$

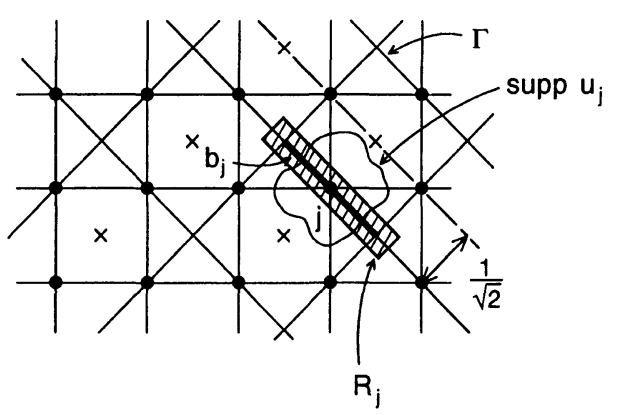

Fig. 2. The region $\mathscr{R}_{j}$ and potential sites. 
Obviously, $\mathscr{R}_{j}$ does not intersect the support of the other single-site potentials centered on $\mathbb{Z}^{2} \backslash\{j\}$ so that $V(x)=\lambda_{j}(\omega) u(x-j) \forall x \in \mathscr{R}_{j}$. Then, if $b_{j}$ is occupied, one has $V(x)<\frac{E-B}{2} \forall x \in \mathscr{R}_{j}$ (recall that $\frac{E-B}{2}>0$ ). We now assume that there is a closed circuit of occupied bonds $\mathscr{C} \equiv \bigcup_{j \in \gamma} b_{j}, \gamma \subset \mathbb{Z}^{2}$ (i.e. a connected union occupied bonds). We call $\mathscr{R} \equiv \bigcup_{j \in \gamma} \mathscr{R}_{j}$ the closed ribbon associated with $\mathscr{C}$. For all $x \in \mathscr{R}$, we have $V(x)<\frac{E-B}{2}$. If we take $a \equiv \frac{E-B}{2}$, then

$$
V(x)+B-E<-a, \quad \forall x \in \mathscr{R} .
$$

The existence of a closed ribbon $\mathscr{R}$ so that $V$ satisfies condition (4.2) is a consequence of the existence of a closed circuit $\mathscr{C}$ in $\Gamma$ of occupied bonds. In order to estimate the probability that $\mathscr{C}$ exists, we use some standard results of percolation theory (see, e.g. [21 and 22]) which we now summarize. For a subset $\mathcal{O} \subset \mathbb{R}^{2}$, the in-radius of $\mathcal{O}$ is defined to be $\sup \left\{R>0 \mid B_{R} \subset \mathcal{O}\right\}$, where $B_{R}$ denotes a ball of radius $R$. We will write $\operatorname{Inrad} \mathcal{O}$ for the in-radius of $\mathcal{O}$.

Let $\mathbb{Z}^{2}$ be the square lattice (the length of the side plays no role in the calculations). A bond (edge) of $\mathbb{Z}^{2}$ is said to be occupied with probability $p, 0 \leqq$ $p \leqq 1$, and empty with probability $1-p$. We are interested in the case when the bonds are independent (called Bernoulli bond percolation). The critical percolation probability $p_{c}$ is defined as follows. Let $P_{\infty}(p)$ be the probability that the origin belongs to an infinite (connected) cluster of occupied bonds. Then, we define

$$
p_{c} \equiv \inf \left\{p \mid P_{\infty}(p)>0\right\} .
$$

For 2-dimensional Bernoulli bond percolation, $p_{c}=\frac{1}{2}$. Hence if $p>p_{c}$, occupied bonds percolate; that is, we can find a connected cluster of occupied bonds running off to infinity with non-zero probability.

Of importance for us are the results concerning the existence of closed circuits of occupied bonds. Let $r_{n, l}$ be a rectangle in $\mathbb{Z}^{2}$ of width $l$ and length $n l$. Let $R_{n, l}$ be the probability that there is a crossing of $r_{n, l}$, the long way, by a connected path of occupied bonds. This probability is controlled by an exponential factor $m(p)$, which is strictly positive for $p<p_{c}$ and $m(p) \searrow 0$ as $p \nearrow p_{c}$. This factor measures the probability that the origin 0 is connected to $x \in \mathbb{Z}^{2}$ by a path of occupied bonds

$$
P_{0 x}(p) \leqq e^{-m(p)|x|} .
$$

The basic result is

Theorem 4.1. For $p>p_{c}, R_{n, l} \geqq 1-C_{0} n l e^{-m(1-p) l}$, for some constant $C_{0}$.

Let us write $r_{l}$ for $r_{1, l}$, the box of side $l$. An annular region between two concentric boxes is denoted by $a_{l} \equiv r_{3 l} / r_{l}$. A closed circuit of occupied bonds in $a_{l}$ is a connected path of occupied bonds lying entirely within $a_{l}$. Using Theorem 4.1 and the $F G K$ inequality, one can compute the probability $A_{l}$ of a closed circuit of occupied bonds in $a_{l}$ for $p>p_{c}$.

Theorem 4.2. For any $p \in[0,1], A_{l} \geqq\left[R_{3, l}(p)\right]^{4}$. In particular, if $p>p_{c}$, $\exists 0<C_{0}<\infty$ as in Theorem 4.1, such that

$$
A_{l} \geqq 1-12 C_{0} l e^{-m(1-p) l} .
$$




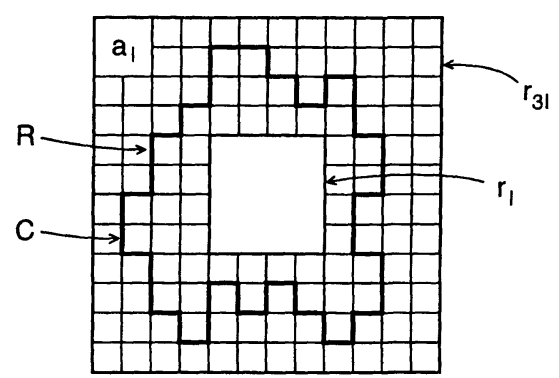

Fig. 3. A closed circuit $\mathscr{C} \subset a_{l}$ and the corresponding ribbon $\mathscr{R}$.

We now apply these results to our situation as follows. On the lattice $\Gamma$ defined above, the probability that any bond is occupied is given by

$$
p=\int_{-M}^{a} g(\lambda) d \lambda
$$

so, under our assumptions on the density $g$, if $a>0$ then $p>p_{c}=\frac{1}{2}$, and we are above the critical percolation threshold $p_{c}=\frac{1}{2}$. Note that when $E=B, a=0$, so $p=\frac{1}{2}=p_{c}$, the critical probability. It follows from Theorem 4.2 that any annular region $a_{l} \equiv r_{3 l} \backslash r_{l}$ in $\Gamma$ of in-radius $\sqrt{2} l \equiv \frac{1}{2}(3 \sqrt{2} l-\sqrt{2} l)$ and sides parallel to the bonds of $\Gamma$ (see Fig. 3 ) contains a closed circuit of occupied bonds with probability given by (4.3). By the argument above, there is a ribbon $\mathscr{R}$ associated with $\mathscr{C}$ in $a_{l}$ whose properties we summarize in the next proposition.

Proposition 4.1. Assume (V1) and (V2) and suppose that $\operatorname{supp} u \subset B_{r_{u}}(0)$. Let $l>\sqrt{2}, E \in \sigma_{0} \backslash\{B\}$, and $a>0$. Then for $m(1-p)$ and $C_{0}$ as in Theorem 4.2, $\exists$ a ribbon $\mathscr{R}$ satisfying

$$
\begin{gathered}
\operatorname{inrad} \mathscr{R} \geqq 2\left(\frac{1}{\sqrt{2}}-r_{u}\right) ; \\
\operatorname{dist}\left(\mathscr{R}, \partial r_{3 l}\right), \operatorname{dist}\left(\mathscr{R}, \partial r_{l}\right) \geqq \frac{1}{\sqrt{2}}+r_{u} ; \quad \mathscr{R} \subset a_{l},
\end{gathered}
$$

and such that

$$
V(x)+B-E<-a, \quad \forall x \in \mathscr{R},
$$

with a probability larger than

$$
1-12 C_{0} l e^{-m(1-p) l}
$$

where

$$
p \equiv \int_{-M}^{a} g(\lambda) d \lambda
$$

4.2. Decay Estimates. The effective one Landau band Hamiltonian $B+V$ localizes electrons at energies $E$ where the equipotential lines $E=V(x)+B$ don't percolate to infinity. The effect of the interband interaction is to induce some tunneling through the "Classically Forbidden" ribbon $\mathscr{R}$ of Proposition 4.1. As a consequence, instead of localization in the compact subsets of $\mathbb{R}^{2}$ bounded by 
$\mathscr{R}$, one expects exponential decay of the Green's function in $x$ and $B$ across such ribbons $\mathscr{R}$. Such an estimate is the starting point of the inductive, multiscale analysis detailed in Sect. 5. By the geometric resolvent equation, we show there that it suffices to consider the following ideal situation, where for some $a>0$,

$$
V(x)+B-E<-a, \quad \forall x \in \mathbb{R}^{2},
$$

or, alternately,

$$
V(x)+B-E>a, \quad \forall x \in \mathbb{R}^{2} .
$$

A condition such as (4.9) with $E>B$ is satisfied, with a probability given in Proposition 4.1, by a smoothing (see Sect. 5) of the potential $V_{\mathscr{R}}$ defined as

$$
V_{\mathscr{R}}(x)= \begin{cases}V(x) & x \in \mathscr{R} \\ 0 & x \in \mathbb{R}^{2} \backslash \mathscr{R} .\end{cases}
$$

Here we obtain decay estimates on

$$
H=H_{A}+V
$$

with $V$ having compact support with non-empty interior and satisfying (4.9) or (4.10).

Let $\mathcal{O}$ be an open, bounded, connected set in $\mathbb{R}^{2}$ with smooth boundary and define $\rho(x)=\operatorname{dist}(x, \mathcal{O})$. Let $\eta \in C_{0}^{\infty}\left(\mathbb{R}^{2}\right)$ with $\eta>0$ and supp $\eta \subset B_{1}(0)$. For any $\varepsilon>0$, define $\eta_{\varepsilon}(x)=\eta(x / \varepsilon)$. We consider the smoothed distance function $\rho_{\varepsilon}(x) \equiv\left(\eta_{\varepsilon} \star \rho\right)(x)$; supp $\rho_{\varepsilon} \subset \mathbb{R}^{2} \backslash\left\{x \mid \operatorname{dist}\left(x, \mathcal{O}^{c}\right)<\varepsilon\right\}$. We fix $\varepsilon>0$ small and write $\rho$ for $\rho_{\varepsilon}$ below for simplicity. We have $\|\nabla \rho\|_{\infty}<C_{0} / \varepsilon$ and $\|\Delta \rho\|_{\infty}<$ $C_{1} / \varepsilon^{2}$, for constants $C_{0}, C_{1}>0$ depending only on $\eta$ and $\mathcal{O}$. This $\varepsilon$ will play no role in the analysis below and, consequently, we absorb it into the constants $C_{0}$ and $C_{1}$. We consider one-parameter families of operators defined for $\alpha \in \mathbb{R}$ as

$$
\begin{gathered}
H_{A}(\alpha) \equiv e^{i \alpha \rho} H_{A} e^{-i \alpha \rho} ; \\
H(\alpha) \equiv H_{A}(\alpha)+V ; \\
P(\alpha) \equiv e^{i \alpha \rho} P e^{-i \alpha \rho}, \text { etc } .
\end{gathered}
$$

Here, we write $P$ for the projector $P_{0}$ and $Q \equiv 1-P$. For $\alpha \in \mathbb{R}$, these families are unitarily equivalent with the $\alpha=0$ operators.

Lemma 4.1. The family $H(\alpha), \alpha \in \mathbb{R}$, has an analytic continuation into the strip

$$
S \equiv\left\{\alpha \in \mathbb{C}|| \operatorname{Im} \alpha \mid<\eta_{\rho} B^{1 / 2}\right\},
$$

as a type A analytic family with domain $D(H)$. The positive constant $\eta_{\rho}$ depends only on the distance function $\rho$. Furthermore, in this strip $S$, one has $P(\alpha)^{2}=P(\alpha)$ and for some constant $C_{1}$ independent of $\alpha$,

$$
\|P(\alpha)\|<C_{1}
$$

and

$$
\left\|Q(\alpha)\left(H_{A}(\alpha)-z\right)^{-1}\right\|<C_{1} B^{-1}, \quad \text { if } \operatorname{dist}(z, B) \leqq B .
$$


Proof. For $\alpha \in \mathbb{R}$, one has

$$
\begin{aligned}
H_{A}(\alpha) & =(-i \nabla-\alpha \nabla \rho-A)^{2} \\
& =H_{A}-\alpha[\nabla \rho \cdot(p-A)+(p-A) \cdot \nabla \rho]+\alpha^{2}|\nabla \rho|^{2} \\
& =H_{A}+\alpha^{2}|\nabla \rho|^{2}+i \alpha \Delta \rho-2 \alpha \nabla \rho \cdot(p-A) .
\end{aligned}
$$

We first show that $H_{A}(\alpha)$ is a type $A$ analytic family on the strip $S$. For this, it suffices to show that

$$
\left\{\alpha^{2}|\nabla \rho|^{2}+i \alpha \Delta \rho-2 \alpha \nabla \rho \cdot(p-A)\right\}\left(H_{A}-z\right)^{-1},
$$

has norm less than 1 for some $z \notin \sigma\left(H_{A}\right)$ and $|\operatorname{Im} \alpha|<\eta_{\rho} B^{1 / 2}$ (cf. [23], in particular, Theorem IV.1.1 and Chapter VII.2). For later purposes, we choose $z \in C(B) \equiv$ $\{z|| z-B \mid=B\}$, circle of radius $B$ centered at $B$. Since $|\nabla \rho|^{2}$ and $\Delta \rho$ are bounded, we can choose $\eta_{\rho}^{\prime}$ such that

$$
\left\|\left(\alpha^{2}|\nabla \rho|^{2}+i \alpha \Delta \rho\right)\left(H_{A}-z\right)^{-1}\right\|<1 / 2,
$$

for $|\alpha|<\eta_{\rho}^{\prime} B^{1 / 2}$ and for all $z \in C(B)$. Hence, it is enough to show that $\forall|\alpha|<\eta_{\rho}^{\prime \prime} B^{1 / 2}$, for a possibly smaller constant $\eta_{\rho}^{\prime \prime}$,

$$
2|\alpha|\left\|\nabla \rho \cdot(p-A)\left(H_{A}-z\right)^{-1}\right\|<1 / 2,
$$

for some $z \in C(B)$. Since $\|\nabla \rho\|_{\infty}<C_{0}$, we easily find that $\| \nabla \rho \cdot(p-A)\left(H_{A}-\right.$ $z)^{-1} \|<C_{1} B^{-1 / 2}$. This implies (4.19) for all $B$ sufficiently large. We take $\eta_{\rho}$ to be the smallest of these two constants. Since type $A$ analyticity is stable under bounded perturbations, it follows that $H(\alpha)$ is a type $A$ analytic family of operators on $S$. From these estimates and (4.18) for $\alpha \in S$, we have that for $B$ large enough,

$$
\left\|\left(H_{A}(\alpha)-z\right)^{-1}\right\|<C_{2} B^{-1}, \quad z \in C(B),
$$

for some constant $C_{2}$ uniform in $\alpha \in S$ and $z \in C(B)$. Next, note that the eigenfunctions of $H_{A}$ are analytic vectors for the family $e^{i \alpha \rho}, \alpha \in S$. It is a consequence of this, the analyticity of $H_{A}$, and the eigenvalue equation, that $\sigma\left(H_{A}(\alpha)\right)$ is independent of $\alpha, \alpha \in S$. The family $P(\alpha), \alpha \in \mathbb{R}$, has an analytic continuation in $S$ given by the contour integral

$$
P(\alpha)=\frac{-1}{2 \pi i} \int_{C(B)}\left(H_{A}(\alpha)-z\right)^{-1} d z .
$$

The boundedness of $P(\alpha)$ follows from (4.20) and (4.21). The idempotent property of $P(\alpha)$ follows from this analyticity and the identity $P(\alpha)^{2}=P(\alpha)$, which holds for real $\alpha$. Furthermore, the function $\alpha \in S \rightarrow Q(\alpha)\left(H_{A}(\alpha)-z\right)^{-1}$ is holomorphic on and inside $C(B)$. By the maximum modulus principle, it follows that

$$
\left\|Q(\alpha)\left(H_{A}(\alpha)-z\right)^{-1}\right\| \leqq \sup _{z \in C(B)}\left\|Q(\alpha)\left(H_{A}(\alpha)-z\right)^{-1}\right\|
$$

and the bound (4.17) follows from this, (4.16) and (4.20).

We next prove the main estimate of this section.

Theorem 4.3. Assume that $(V, E, B)$ satisfy (4.9) or (4.10) for some $a>0$ and $E \in \sigma_{0} \backslash\{B\}$. Furthermore, assume that supp $V$ is compact with non-empty interior. There exists constants $C_{2} \leqq \eta_{\rho}, C_{3}$, and $B_{1}$, depending only on $M_{0} \equiv\|V\|_{\infty}$, 
$\|\nabla \rho\|_{\infty}$, and $\|\nabla V\|_{\infty}$, such that if we define $\gamma \equiv C_{2} \min \left\{B^{1 / 2}, a B\right\}$, and $u$ is a solution of

$$
\left(H_{A}+V-z\right) u=v, \quad z \equiv E+i \varepsilon, \varepsilon>0, E>0,
$$

for some $v \in D\left(e^{\gamma \rho}\right)$, then for $B>B_{1}, \forall \alpha \in \mathbb{C},|\operatorname{Im} \alpha|<\gamma$, we have

$$
\begin{gathered}
u \in D\left(e^{i \rho \alpha}\right), \\
\left\|e^{i \alpha \rho} P u\right\| \leqq C_{3} a^{-1}\left\|e^{i \alpha \rho} v\right\|,
\end{gathered}
$$

and

$$
\left\|e^{i \alpha \rho} Q u\right\| \leqq C_{3} B^{-1}\left\|e^{i \alpha \rho} v\right\| .
$$

Proof. Let $v(\alpha)=e^{i \alpha \rho} v$, so that $v(\alpha)$ is analytic in the strip $|\operatorname{Im}(\alpha)|<\gamma$. Let $u(\alpha)=e^{i \alpha \rho} u, \alpha \in \mathbb{R}$, i.e.,

$$
u(\alpha)=(H(\alpha)-z)^{-1} v(\alpha) \equiv\left((H-z)^{-1} v\right)(\alpha),
$$

with $H \equiv H_{A}+V$, as above. Since $V$ is $H_{A}$-compact by assumption, $H$ has point spectrum, and according to Lemma 4.1 and standard arguments, $H(\alpha)$ has real spectrum independent of $\alpha$ in the strip $S$ defined in (4.15). Then, $u(\alpha)$ has an analytic continuation in $|\operatorname{Im}(\alpha)|<\gamma$, which proves (4.24). Furthermore, this continuation satisfies (4.27) in the whole strip $S$, i.e. $(H(\alpha)-z) u(\alpha)=v(\alpha)$, for all $\alpha \in S$. Projecting this equation along $P(\alpha)$ gives

$$
(B+V-z)(P u)(\alpha)=(P v)(\alpha)+([Q V P-P V Q] u)(\alpha),
$$

where $(P u)(\alpha) \equiv P(\alpha) u(\alpha)$, etc. Taking the scalar product of (4.28) with $(P u)(\alpha)$ results in the inequality,

$$
\begin{aligned}
a\|(P u)(\alpha)\|^{2} \leqq & \|(P u)(\alpha)\|\|(P v)(\alpha)\| \\
& +\left\{\left\|\left(P^{*} Q\right)(\alpha)\right\|\|(Q V P)(\alpha)\|\right\}\|(P u)(\alpha)\|^{2} \\
& +\|(P V Q)(\alpha)\|\|(P u)(\alpha)\|\|(Q u)(\alpha)\| .
\end{aligned}
$$

In the appendix, we prove that for $B$ large enough,

$$
\|(Q V P)(\alpha)\| \leqq C_{4} B^{-1 / 2},
$$

and

$$
\left\|\left(P^{*} Q\right)(\alpha)\right\| \leqq C_{5}|\operatorname{Im} \alpha| B^{-1 / 2} .
$$

With these estimates, we obtain from (4.29),

$$
\begin{aligned}
\left(a-C_{6} \gamma B^{-1}\right)\|(P u)(\alpha)\|^{2} \leqq & \|(P u)(\alpha)\|\|(P v)(\alpha)\| \\
& +C_{7} B^{-1 / 2}\|(P u)(\alpha)\|\|(Q u)(\alpha)\|,
\end{aligned}
$$

where the constants $C_{6}$ and $C_{7}$ depend only on $\|V\|_{\infty},\|\nabla V\|_{\infty}$, and $\|\nabla \rho\|_{\infty}$. To estimate $\|(Q u)(\alpha)\|$, it follows from the resolvent equation and (4.27) that

$$
\begin{aligned}
\|(Q u)(\alpha)\| \leqq & \left\|\left(Q\left(H_{A}-z\right)^{-1} v\right)(\alpha)\right\|+\left\|\left\{Q\left(H_{A}-z\right)^{-1} Q V(Q+P) u\right\}(\alpha)\right\| \\
\leqq & C_{1} B^{-1}\|v(\alpha)\|+C_{1} B^{-1} M_{0}\|(Q u)(\alpha)\| \\
& +C_{1} B^{-1} M_{0}\|(Q V P u)(\alpha)\|,
\end{aligned}
$$


with $M_{0} \equiv\|V\|_{\infty}<\infty$. Using the estimate on $Q V P$ derived in the appendix and taking $B>2 M_{0} C_{1}$, we obtain,

$$
\|(Q u)(\alpha)\| \leqq 2 C_{1} B^{-1}\|v(\alpha)\|+C_{8} B^{-3 / 2}\|(P u)(\alpha)\|,
$$

where $C_{8} \equiv 2 M_{0} C_{1} C_{2}$. Substituting (4.32) into (4.30), we obtain

$$
\left(a-C_{6} \gamma B^{-1}-C_{7} C_{8} B^{-2}\right)\|(P u)(\alpha)\| \leqq\left(C_{1}+2 C_{1} C_{7} B^{-3 / 2}\right)\|v(\alpha)\| .
$$

This proves (4.25) for $B$ large enough. Inserting (4.25) into (4.32) yields (4.26).

Corollary 4.1. Let $\mathcal{O}$ be an open, connected, bounded subset of $\mathbb{R}^{2}$ with smooth boundary and suppose $\mathscr{E} \subset \mathbb{R}^{2} \backslash \mathcal{O}$. Let $E \in \sigma_{0} \backslash\{B\}$ and assume that $(B, E, V)$ satisfy (4.9) or (4.10) for some $a>0$. Let $\chi_{X}, X=\mathcal{O}$ and $\mathscr{E}$, be bounded functions with support in $X$ and s.t. $\left\|\chi_{X}\right\|_{\infty} \leqq 1$. Then,

$$
\sup _{\varepsilon \neq 0}\left\|\chi_{\mathscr{E}}\left(H_{A}+V-E-i \varepsilon\right)^{-1} \chi_{\mathcal{O}}\right\| \leqq C_{3} \max \left\{a^{-1}, B^{-1}\right\} e^{-\gamma d}
$$

where $C_{3}$ and $\gamma$ are as in Theorem 4.3 and $d \equiv \operatorname{dist}(\mathcal{O}, \mathscr{E})$.

Proof. This is an immediate consequence of Theorem 4.3. We set $\rho(x) \equiv \operatorname{dist}(x, \mathcal{O})$ and choose $v \equiv \chi_{\mathcal{O}} v$. Then, $e^{i \alpha \rho} v=v, \forall \alpha \in \mathbb{C}$. For $u$ a solution of $\left(H_{A}+V-E-\right.$ $i \varepsilon) u=\chi_{\mathcal{O}} v$, one has $\forall \alpha \in \mathbb{C},|\operatorname{Im} \alpha|<\gamma$,

$$
\begin{aligned}
\left\|\chi_{\mathscr{E}}\left(H_{A}+V-E-i \varepsilon\right)^{-1} \chi_{\mathcal{O}} v\right\| & =\left\|\chi_{\mathscr{E}}(P+Q) u\right\| \leqq e^{-d(\operatorname{Im} \alpha)}\left\{\left\|e^{-i \alpha \rho} P u\right\|+\left\|e^{-i \alpha \rho} Q u\right\|\right\} \\
& \leqq e^{-d(\operatorname{Im} \alpha)} C_{3} \max \left\{a^{-1}, B^{-1}\right\}\|v\|
\end{aligned}
$$

by Theorem 4.3. Taking $\operatorname{Im} \alpha \rightarrow \gamma$, we obtain (4.34).

\section{Proof of the Main Theorem}

We will show below that Corollary 4.1 implies hypothesis [H1] $\left(\gamma_{0}, l_{0}\right)$ of [8]. This hypothesis, along with Wegner's estimate, Theorem 3.1, are the main starting points of the multi-scale analysis described in [8]. The goal of this analysis is to verify the main assumption (A2) of Theorem 3.2 of [8], which gives sufficient conditions for pure point spectrum in an interval and exponential decay of eigenfunctions. A version of Kotani's trick, necessary to control the singular continuous spectrum for the models studied here, follows from Lemma 3.2.

In order to make this paper more self-contained, we recall the main points of this analysis here and refer to [8] for the details. To reduce the family $H_{\omega}$ in (2.1) to a one parameter family, we consider variations $\omega^{\prime} \in \Omega$ for which only $\lambda_{0}$ changes. For $\omega$ fixed and $\lambda \equiv \lambda_{0}\left(\omega^{\prime}\right)-\lambda_{0}(\omega)$, we have

$$
H_{\omega^{\prime}}=H_{\omega}+\lambda u \equiv H_{0}+\lambda u \equiv H_{\lambda}(A)
$$

with $u$ satisfying $(\mathrm{V} 1)$. Let $R_{\lambda}(z) \equiv\left(H_{\lambda}-z\right)^{-1}$. We first check the compactness condition, (A1), of [8]. By the diamagnetic inequality,

$$
e^{-H_{\lambda}(A) t} \leqq e^{-H_{\lambda}(0) t}, \quad t \geqq 0,
$$


and it is clear that $u^{1 / 2} e^{-H_{\lambda}(0) t} u^{1 / 2}, t>0$, is compact. Using the integral representation

$$
R_{\lambda}(x)=\int_{0}^{\infty} e^{-\left(H_{\lambda}(A)-x\right) t} d t, \quad x<0,
$$

it follows from the norm-convergence of the integral and inequality (5.2) that $u^{1 / 2} R_{\lambda}(z) u^{1 / 2}$ is compact for $\operatorname{Im} z \neq 0, \forall \lambda$. This, for $\lambda=0$, is condition (A1) of [8].

The second condition (A2) is that $\exists I_{0} \subset I, I$ some interval, and $\left|I_{0}\right|=|I|$ s.t. $\forall E \in I_{0}$,

$$
\sup _{\varepsilon \neq 0}\left\|R_{0}(E+i \varepsilon) u^{\frac{1}{2}}\right\|<\infty .
$$

The multi-scale analysis is used to verify this condition for a.e. $\omega$ (recall $H_{0}=H_{\omega}$ ). The main theorem, which we recall in the present context, concerning condition (5.4), is the following.

Theorem 5.1. (Theorem 2.3 of [8]). Let $\gamma_{0}>0 . \exists$ a minimum length scale $l^{\star} \equiv l^{\star}\left(\gamma_{0}, C_{W}\right)$, s.t.: if $[\mathrm{H} 1]\left(\gamma_{0}, l_{0}\right)$ holds at energy $E$ for $l_{0}>l^{\star}$, then for $\mathbb{P}$-a.e. $\omega \exists$ a finite constant $d_{\omega}>0$ s.t.

$$
\sup _{\varepsilon \neq 0}\left\|\left(H_{\omega}-E-i \varepsilon\right)^{-1} u^{\frac{1}{2}}\right\|<d_{\omega} \delta(u)
$$

where $\delta(u)$ depends only on $u$.

We prove below that $[\mathrm{H} 1]\left(\gamma_{0}, l_{0}\right)$ holds at each energy in $\left[B-M_{0}, B-\right.$ $\left.\mathcal{O}\left(B^{-1}\right)\right] \cup I_{0}(B) \cap \sigma_{0}$ with a suitable $\gamma_{0}$ (see Proposition 5.1) and for all $l_{0}$ large enough provided $B$ is large. By Theorem 3.2 of [8], this theorem and the compactness result shown above imply that $H_{\lambda}$ in (5.1) has pure point spectrum in this set for a.e. $\lambda$. By the probabilistic arguments of [8], we conclude that $H_{\omega}$ has only pure point spectrum in this set for $\mathbb{P}$-a.e. $\omega$.

The second main theorem which we recall here allows us to prove exponential decay of the eigenfunctions.

Theorem 5.2. (Theorem 2.4 of [8]). Let $\chi_{x}$ be the characteristic function of a unit cube centered at $x \in \mathbb{R}^{2}$. Under the assumptions of Theorem 5.1, for $\mathbb{P}$-a.e. $\omega \exists$ a finite constant $d_{\omega}>0$ s.t. for all $x,\|x\|$ large enough,

$$
\sup _{\varepsilon>0}\left\|\chi_{x}\left(H_{\omega}-E-i \varepsilon\right)^{-1} u^{\frac{1}{2}}\right\| \leqq d_{\omega} e^{-\gamma_{1}\|x\|}
$$

where $\gamma_{1} \equiv(1 / 6 \sqrt{2}) \gamma_{0}, \gamma_{0}$ as in Theorem 5.1.

Let us remark that for our problem, $\gamma_{0} \sim B^{\sigma}$, for some $\sigma>0$ so there is exponential decay in the $B$-field also. We now turn to the proof of $[\mathrm{H} 1]\left(\gamma_{0}, l_{0}\right)$.

To begin, we introduce some geometry. In this section, we work with subregions of the lattice $\Gamma \equiv e^{i \pi / 4} \sqrt{2} \mathbb{Z}^{2}$, introduced in Sect. 4 , rather than in $\mathbb{Z}^{2}$. Recall that there is a $1: 1$ correspondence between bonds $b_{j} \in \Gamma$ and vertices $j \in \mathbb{Z}^{2}$. We arbitrarily choose a vertex of $\Gamma$ as the origin and take coordinate axes parallel to the bonds of $\Gamma$. We define boxes $\Lambda_{l} \subset \Gamma$ relative to this point,

$$
\Lambda_{l} \equiv\left\{x \in \mathbb{R}^{2}|| x_{i} \mid \leqq l / 2 \text { for } i=1,2\right\} .
$$


For convenience, we fix points so the bond $b_{0}$ has one of its ends at $0 \in \Gamma$. For any $\delta>0$, consider $\Lambda_{l, \delta} \equiv\left\{x \in \Lambda_{l} \mid \operatorname{dist}\left(x, \partial \Lambda_{l}\right)<\delta\right\}$. Let $\chi_{l, \delta}$ be the $C^{2}$-function which satisfies $\operatorname{supp} \chi_{l, \delta} \subset \Lambda_{l}, \chi_{l, \delta} \geqq 0,\left|\nabla \chi_{l, \delta}\right| \subset \Lambda_{l} \backslash \Lambda_{l, \delta}$ and $\chi_{l, \delta} \mid \Lambda_{l, \delta}=1$. Let $W(\chi) \equiv\left[\chi, H_{A}\right]$, for any $\chi \in C^{2}$.

As described in the beginning of Sect. 3, we work with the modified local Hamiltonians $\hat{H}_{A}$ in order to utilize the independence of the potentials and (3.1). Let $\Lambda \subset \mathbb{R}^{2}$ be a rectangular region with sides parallel to the bonds of $\Gamma$ and corners at the vertices of $\Gamma$. We define a slightly smaller subrectangle $\hat{\Lambda} \subset \Lambda$, with sides parallel to the bonds of $\Gamma$, by $\hat{\Lambda} \equiv \Lambda /\{x \in \Lambda \mid\|x-y\|<1 / \sqrt{2} \forall y \in \partial \Lambda\}$. We define a local potential associated with $\Lambda$ to be $\hat{V}_{\Lambda} \equiv \sum_{i \in \hat{\Lambda} \cap \Gamma} \lambda_{i}(\omega) u(x-i)$ and the local Hamiltonian $\hat{H}_{\Lambda} \equiv H_{A}+\hat{V}_{\Lambda}$. The Wegner estimate, Theorem 3.1, and the results of Sect. 4 , hold for $\hat{H}_{\Lambda}$. For simplicity of notation, we will continue to write $H_{\Lambda}$ for $\hat{H}_{\Lambda}$ below.

We apply the multi-scale analysis to $H_{\Lambda}$ relative to the lattice $\Gamma$. We verify condition [H1] $\left(\gamma_{0}, l_{0}\right)$ of [8] using Corollary 4.1 and the geometric resolvent equation $(G R E)$. We must show that for $E \in\left[B-M_{0}, B-\mathcal{O}\left(B^{-1}\right)\right] \cup I_{0}(B) \cap \sigma_{0}$ and for all $l_{0}$ sufficiently large, that the following holds:

$[\mathrm{H} 1]\left(\gamma_{0}, l_{0}\right)$ For some $\gamma_{0}>0, l_{0}>1, \exists \xi>4$ s.t.

$$
\mathbb{P}\left\{\sup _{\varepsilon>0}\left\|W\left(\chi_{l, \delta}\right) R_{\Lambda_{l_{0}}}(E+i \varepsilon) \chi_{l_{0 / 3}}\right\| \leqq e^{-\gamma_{0} l_{0}}\right\} \geqq 1-l_{0}^{-\xi} .
$$

We begin with a simple lemma which allows us to control the gradient term in $W\left(\chi_{l, \delta}\right)$.

Lemma 5.1. Let $H_{\Lambda} \equiv(p-A)^{2}+V_{\Lambda}$ and write $R_{\Lambda} \equiv R_{\Lambda}(E+i \varepsilon), \varepsilon \neq 0, E \in \mathbb{R}$. For any $u \in L^{2}\left(\mathbb{R}^{2}\right),\|u\|=1$, we have for $i=1,2$,

$$
\left\|(p-A)_{i} R_{\Lambda} u\right\|^{2} \leqq\left\|R_{\Lambda} u\right\|+\left(2 M_{0}+|E|\right)\left\|R_{\Lambda} u\right\|^{2},
$$

where $M_{0} \equiv\left\|V_{\Lambda}\right\|_{\infty}>0$. Moreover, for any bounded $\chi \in C^{1}$, we have,

$$
\begin{aligned}
\sum_{i=1}^{2}\left\|\chi(p-A)_{i} R_{\Lambda} u\right\|^{2} \leqq & \left\|\chi R_{\Lambda} u\right\|\|\chi u\|+\left(2 M_{0}+|E|\right)\left\|\chi R_{\Lambda} u\right\|^{2} \\
& +2 \sum_{i=1}^{2}\left\|\left(\partial_{i} \chi\right) R_{\Lambda} u\right\|\left\|\chi(p-A)_{i} R_{\Lambda} u\right\|
\end{aligned}
$$

Proof. The inequality (5.5) follows directly from the equality

$$
\left\langle R_{\Lambda} u, H_{A} R_{\Lambda} u\right\rangle=\left\langle R_{\Lambda} u, u\right\rangle-\left\langle R_{\Lambda} u,\left(V_{\Lambda}-E-i \varepsilon\right) R_{\Lambda} u\right\rangle,
$$

and the Cauchy-Schwartz inequality. The inequality (5.6) follows in the same way by writing out $\left\|\chi(p-A)_{i} R_{\Lambda} u\right\|^{2}$.

We now prove the main result of this section. Recall that $\mathscr{R}$ denotes the ribbon defined in Sect. 4.

Proposition 5.1. Let $\chi_{2}$ be any function, $\left\|\chi_{2}\right\|_{\infty} \leqq 1$, supported on $\Lambda_{l} \cap$ Ext $\mathscr{R}$, where Ext $\mathscr{R} \equiv\left\{x \in \mathbb{R}^{2} \mid \lambda x \notin \mathscr{R} \forall \lambda \geqq 1\right\}$, so that, in particular, supp $\chi_{2} \cap \mathscr{R}=\emptyset$. 
For any $E \in \sigma_{0} \backslash\{B\}, \delta>0, \varepsilon>0$, and $a>0$, we have

$$
\sup _{\varepsilon \neq 0}\left\|\chi_{2} R_{\Lambda_{l}}(E+i \varepsilon) \chi_{l / 3}\right\| \leqq C e^{-\gamma d} \max \left\{a^{-1}, B^{-1}\right\} \cdot \max \left\{\delta^{-1},\left(2 M_{0}+|E|\right) \delta^{-2}\right\}
$$

where $C$ depends on $C_{3}$ of Theorem 4.3 and $\chi_{2}, \gamma$ is defined in Theorem 4.3, and $d \equiv\left(r_{1}-3 \varepsilon\right) / 2\left(r_{1} \equiv \operatorname{inrad} \mathscr{R}\right)$, with a probability larger than

$$
1-\left\{C l e^{-m l}+C_{W}[\operatorname{dist}(E, B)-\delta]^{-2}\|g\|_{\infty} \delta B l^{2}\right\} .
$$

In particular, for $\chi_{l, \delta}$ defined above and $E \in \sigma_{0}$ with $a=\frac{E-B}{2}=\mathcal{O}\left(B^{-1+\sigma}\right)$, any $\sigma>0$, we have that for any $l_{0}>\sqrt{2}$ and large enough, and any $\xi>4, \exists B\left(l_{0}\right)>0$ s.t. $\forall B>B\left(l_{0}\right),[\mathrm{H1}]\left(\gamma_{0}, l_{0}\right)$ holds for some $\gamma_{0}>\gamma d / 4 l_{0}>0$, so that $\gamma_{0}=$ $\mathcal{O}\left\{\min \left(B^{1 / 2}, B^{\sigma}\right)\right\}$.

Proof. 1. By Proposition 4.1, $\exists B_{0}$ s.t. $B>B_{0}$ implies $\exists$ a ribbon $\mathscr{R} \subset \Lambda_{l} \backslash \Lambda_{l / 3}$ (with a probability given by (4.7)) satisfying

$$
\operatorname{dist}\left(\mathscr{R}, \partial \Lambda_{l}\right), \quad \text { and } \quad \operatorname{dist}\left(\mathscr{R}, \partial \Lambda_{l / 3}\right)>\frac{1}{\sqrt{2}}+r_{u}>0 \text {, }
$$

and

$$
r_{1} \equiv \operatorname{inrad} \mathscr{R}>2\left(\frac{1}{\sqrt{2}}-r_{u}\right)
$$

and such that

$$
V(x)+B-E>-a, \quad \forall x \in \mathscr{R}, a=\frac{E-B}{2} .
$$

(We assume $E>B$; similar arguments hold for $E<B$.) For any $\varepsilon>0,3 \varepsilon \ll r_{1}$, define the border of $\mathscr{R}$ by

$$
\mathscr{R}_{\varepsilon} \equiv\{x \in \mathscr{R} \mid \operatorname{dist}(x, \partial \mathscr{R})<\varepsilon\} .
$$

Then $\mathscr{R}_{\varepsilon} \equiv \mathscr{R}_{\varepsilon}^{+} \cup \mathscr{R}_{\varepsilon}^{-}$, where $\mathscr{R}_{\varepsilon}^{ \pm}$are two disjoint, connected subsets of $\mathscr{R}$. Let $\mathscr{C} \equiv\left\{x \in \mathscr{R} \mid \operatorname{dist}\left(x, \mathscr{R}_{\varepsilon}^{+}\right)=\operatorname{dist}\left(x, \mathscr{R}_{\varepsilon}^{-}\right)\right\} ; \mathscr{C}$ is a closed, connected path in $\mathscr{R}$. Let $\mathscr{C}_{\varepsilon} \equiv\{x \in \mathscr{R} \mid \operatorname{dist}(x, \mathscr{C})<\varepsilon / 2\} \subset \mathscr{R}$, so that inrad $\mathscr{C}_{\varepsilon}=\varepsilon$ and

$$
\operatorname{dist}\left(\mathscr{C}_{\varepsilon}, \mathscr{R}_{\varepsilon}^{ \pm}\right) \geqq\left(r_{1}-3 \varepsilon\right) / 2 .
$$

This is strictly positive. Because of this, we can adjust $\mathscr{C}_{\varepsilon}$ so that $\partial \mathscr{C}_{\varepsilon}$ is smooth. We need two, $C^{2}$, positive cut-off functions. Let $\chi_{\mathscr{R}}>0$ satisfy $\chi_{\mathscr{R}} \mid \mathscr{C}_{\varepsilon}=1$ and $\operatorname{supp}\left|\nabla \chi_{\mathscr{R}}\right| \subset \mathscr{R}_{\varepsilon}$. Let $\chi_{1}$ satisfy $\chi_{1} \mid \Lambda_{l / 3}=1$ and $\operatorname{supp}\left|\nabla \chi_{1}\right| \subset \mathscr{C}_{\varepsilon}$ (see Fig. 4). By simple commutation, we have (with $\chi_{2}$ as in the proposition),

$$
\begin{aligned}
\chi_{2} R_{\Lambda_{l}}(E+i \varepsilon) \chi_{l / 3} & =\chi_{2} R_{\Lambda_{l}} \chi_{1} \chi_{l / 3}=\chi_{2} R_{\Lambda_{l}} W\left(\chi_{1}\right) R_{\Lambda_{l}} \chi_{l / 3} \\
& =\chi_{2} R_{\Lambda_{l}} \chi_{\mathscr{R}} W\left(\chi_{1}\right) R_{\Lambda_{l}} \chi_{l / 3} .
\end{aligned}
$$

Next, denote by $R_{\mathscr{R}}$ the resolvent of $H_{\mathscr{R}}$ defined in Sect. 4.2. The $G R E$ relating $R_{\Lambda_{l}}$ and $R_{\mathscr{R}}$ is

$$
R_{\Lambda_{l}} \chi_{\mathscr{R}}=\chi_{\mathscr{R}} R_{\mathscr{R}}+R_{\Lambda_{l}} W\left(\chi_{\mathscr{R}}\right) R_{\mathscr{R}} .
$$




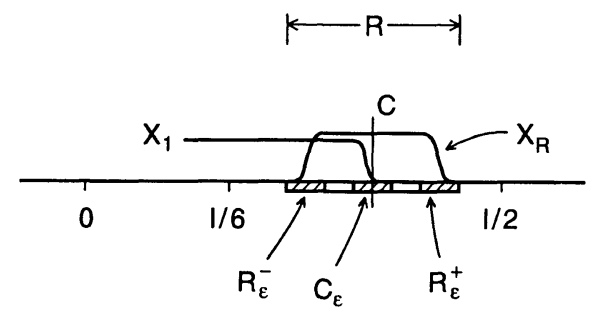

Fig. 4. The ribbon $\mathscr{R}$ and cut-off functions.

Substituting (5.13) into (5.12) and noting that $\chi_{2} \chi_{\mathscr{R}}=0$, we obtain

$$
\chi_{2} R_{\Lambda_{l}} \chi_{l / 3}=\chi_{2} R_{\Lambda_{l}} W\left(\chi_{\mathscr{R}}\right) R_{\mathscr{R}} W\left(\chi_{1}\right) R_{\Lambda_{l}} \chi_{l / 3} .
$$

Note that from (5.12) and the choice of $\chi_{\mathscr{R}}$ and $\chi_{1}$, we obtain that

$$
\operatorname{dist}\left(\operatorname{supp} W\left(\chi_{\mathscr{R}}\right), \operatorname{supp} W\left(\chi_{1}\right)\right) \geqq\left(r_{1}-3 \varepsilon\right) / 2 \text {. }
$$

We apply Wegner's estimate, Theorem 3.1, to control the two $R_{\Lambda_{l}}$ factors in (5.14), and the decay estimate, Corollary 4.1 , to control the factor $R_{\mathscr{R}}$, which is possible due to the localization of $W\left(\chi_{\mathscr{R}}\right)$ and $W\left(\chi_{1}\right)$ and (5.16).

2. To estimate the $R_{\mathscr{R}}(E+i \varepsilon)$ contribution, we use Corollary 4.1 with $\mathcal{O} \equiv \mathscr{C}_{\varepsilon}$ and $\mathscr{E}=\mathscr{R}_{\varepsilon}$. Let $\chi_{X}, X=\mathcal{O}$ and $\mathscr{E}$, be a characteristic function on these sets. Then $W\left(\chi_{\mathscr{R}}\right) \chi_{\mathscr{E}}=W\left(\chi_{\mathscr{R}}\right)$ and $\chi_{\mathscr{O}} W\left(\chi_{1}\right)=W\left(\chi_{1}\right)$. Inserting these localization functions into (5.15), we obtain from Corollary 4.1,

$$
\left\|\chi_{\mathscr{E}} R_{\mathscr{R}}(E+i \varepsilon) \chi_{\mathcal{O}}\right\| \leqq C \max \left\{a^{-1}, B^{-1}\right\} e^{-\gamma d},
$$

with probability larger than

$$
1-C l e^{-m l},
$$

for some $m=m(1-p)>0$ and $0<C<\infty$. The factor $d$ satisfies

$$
d \geqq\left(r_{1}-3 \varepsilon\right) / 2,
$$

where $r_{1} \equiv \operatorname{inrad} \mathscr{R}$ as in (5.10).

3. Next, we turn to

$$
W\left(\chi_{1}\right) R_{l}(E+i \varepsilon) \chi_{l / 3},
$$

and

$$
\chi_{2} R_{l}(E+i \varepsilon) W\left(\chi_{\mathscr{R}}\right),
$$

where we write $R_{l}$ for $R_{\Lambda_{l}}$ for short. We will bound $R_{l}$ by Wegner's estimate and the terms (5.20)-(5.21) via Lemma 5.1. From Theorem 3.1, we have for any $\delta>0$,

$$
\left\|R_{l}(E+i \varepsilon)\right\|<\delta^{-1},
$$

with probability larger than

$$
1-C_{W}[\operatorname{dist}(E, B)-\delta]^{-2}\|g\|_{\infty} \delta B l^{2} .
$$

From (5.22) and Lemma 5.1, both (5.20) and (5.21) are bounded above by

$$
C_{4} \max \left\{\delta^{-\frac{1}{2}},(2 M+|E|)^{\frac{1}{2}} \delta^{-1}\right\},
$$

with probability at least (5.23). The constant $C_{4}$ depends on $\partial^{\alpha} \chi_{1}$, for $|\alpha|=0,1,2$. 
4. Using the estimate $P(A \cap B) \geqq P(A)+P(B)-1$, and (5.17)-(5.18) and (5.23)-(5.24), we find

$$
\left\|\chi_{2} R_{l}(E+i \varepsilon) \chi_{l / 3}\right\| \leqq 2 C \max \left\{a^{-1}, B^{-1}\right\} \cdot \max \left\{\delta^{-1},\left(2 M_{0}+|E|\right) \delta^{-2}\right\} \cdot e^{-\gamma d},
$$

with probability at least

$$
1-\left\{C l e^{-m l}+C_{W}[\operatorname{dist}(E, B)-\delta]^{-2}\|g\|_{\infty} \delta B l^{2}\right\} .
$$

This proves the first part of the proposition.

5. To estimate $W\left(\chi_{l, \delta}\right) R_{l} \chi_{l / 3}$, we use the second formula of Lemma 5.1, (5.6), which gives

$$
\begin{aligned}
\left\|\chi_{2}(p-A)_{i} R_{l} \chi_{l / 3}\right\|^{2} \leqq & \left\|\chi_{2} R_{l} \chi_{l / 3}\right\|+(2 M+|E|)\left\|\chi_{2} R_{l} X_{l / 3}\right\|^{2} \\
& +2 \max _{i=1,2}\left\|\left(\partial_{i} \chi_{2}\right) R_{l} \chi_{l / 3}\right\|\left\|\chi_{2}(p-A)_{i} R_{l} \chi_{l / 3}\right\| .
\end{aligned}
$$

Since $\partial_{i} \chi_{2}$ satisfies the same condition as $\chi_{2}$, the factor $\left\|\left(\partial_{i} \chi_{2}\right) R_{l} \chi_{l / 3}\right\|$ in (5.27) satisfies the estimate (5.24) with possibly a different constant. Solving the quadratic inequality (5.27), we obtain

$$
\begin{aligned}
\left\|\chi_{2}(p-A)_{i} R_{l} \chi_{l / 3}\right\|= & \max _{i=1,2}\left\{\left\|\left(\partial_{i} \chi_{2}\right) R_{l} \chi_{l / 3}\right\|+\left[\left\|\left(\partial_{i} \chi_{2}\right) R_{l} \chi_{l / 3}\right\|^{2}+\left(\left\|\chi_{2} R_{l} \chi_{l / 3}\right\|\right.\right.\right. \\
& \left.\left.\left.+\left(2 M_{0}+|E|\right)\left\|\chi_{2} R_{l} \chi_{l / 3}\right\|^{2}\right)\right]^{1 / 2}\right\}
\end{aligned}
$$

which can be estimated as in (5.25). Finally, we write

$$
\left\|W\left(\chi_{l, \delta}\right) R_{l} \chi_{l / 3}\right\| \leqq\left\|\left(\Delta \chi_{l, \delta}\right) R_{l} \chi_{l / 3}\right\|+2 \sum_{j=1}^{2}\left\|\left(\partial_{j} \chi_{l, \delta}\right)(p-A)_{j} R_{l} \chi_{l / 3}\right\|,
$$

which can be estimated from (5.25) with $\chi_{2} \equiv \Delta \chi_{l, \delta}$ and (5.27) with $\chi_{2} \equiv\left(\partial_{j} \chi_{l, \delta}\right)$.

6. We now show that for any $l_{0}$ large enough, $\exists B_{0} \equiv B_{0}\left(l_{0}\right)$ such that for all $B>B_{0}$, condition [H1] $\left(l_{0}, \gamma_{0}\right)$ is satisfied with $\gamma_{0}=\mathcal{O}\left\{\min \left(B^{1 / 2}, B^{\sigma}\right)\right\}$. We take $E \in\left[B-M_{0}, B-\mathcal{O}\left(B^{-1}\right)\right] \cup I_{0}(B) \cap \sigma_{0}$ and $a=\frac{E-B}{2}=\mathscr{O}\left(B^{-1+\sigma}\right)$, for any $\sigma>0$. First, we require that (5.25) be bounded above by $e^{-\gamma d / 2}$. This leads to the condition

$$
C \delta^{-2} B^{2-\sigma} e^{-\gamma d} \leqq e^{-\gamma d / 2},
$$

where $\gamma=C_{2} \min \left\{B^{\frac{1}{2}}, B^{\sigma}\right\}$. This condition implies that we must choose $\delta$ in (5.22) to satisfy

$$
\delta>B^{1-(\sigma / 2)} e^{-\gamma d / 4} .
$$

If we now define

$$
\gamma_{0} \equiv \gamma d / 4 l_{0}
$$

we find that

$$
\left\|W\left(\chi_{l, \delta}\right) R_{l} \chi_{l / 3}\right\| \leqq e^{-\gamma_{0} l_{0}} .
$$

Next, the probability estimate (5.26) leads to the condition

$$
C l_{0} e^{-m l_{0}}+C_{2} B^{3-2 \sigma} \delta l_{0}^{2} \leqq l_{0}^{-\xi},
$$


or, for all $l_{0}$ large,

$$
C_{3} B^{3-2 \sigma} \delta l_{0}^{2} \leqq l_{0}^{-\xi}
$$

for some $\xi>4$. We can choose $\delta$ so that both conditions (5.31) and (5.33) are satisfied provided the condition

$$
l_{0}^{\xi+2}<B^{3 / 2-(5 / 2) \sigma} e^{\gamma d / 4},
$$

is satisfied for some $\xi>4$. It is clear from the definition of $\gamma$, that for any $l_{0}$, there exists a $B_{0} \equiv B_{0}\left(l_{0}\right)$ such that condition (5.34) is satisfied for all $B>B_{0}$. This completes the proof of the theorem.

\section{Appendix 1}

The following estimates hold for all $B$ sufficiently large.

Lemma A.1. Let $V \in C_{b}^{2}(\mathbb{R}), \exists$ constant $C>0$ depending only on $\left\|\partial^{\alpha} V\right\|_{\infty}$, $|\alpha|=0,1,2$ such that $\forall \alpha \in S$,

$$
\|P(\alpha) V Q(\alpha)\| \leqq C B^{-1 / 2} .
$$

Proof. Let $z \equiv B-1$, so $z \in \rho\left(H_{A}(\alpha)\right)$ for $\alpha \in S$. We have

$$
\begin{aligned}
P(\alpha) V Q(\alpha)= & P(\alpha)\left(H_{A}(\alpha)-z\right)\left(H_{A}(\alpha)-z\right)^{-1} V Q(\alpha) \\
= & P(\alpha)\left(H_{A}(\alpha)-z\right) V\left(H_{A}(\alpha)-z\right)^{-1} Q(\alpha)+P(\alpha)\left(H_{A}(\alpha)-z\right) \\
& \times\left(H_{A}(\alpha)-z\right)^{-1}\left[V, H_{A}(\alpha)\right]\left(H_{A}(\alpha)-z\right)^{-1} Q(\alpha) .
\end{aligned}
$$

Recall that $P(\alpha)$ is analytic in $\alpha \in S$. As

$$
P(\alpha)\left(H_{A}(\alpha)-z\right)=P(\alpha)(B-z)=P(\alpha),
$$

for $\alpha \in \mathbb{R}$, the identity principle for analytic functions implies this holds for $\alpha \in S$. This result (6.3) and estimates (4.16)-(4.17) imply that the first term on the right in (6.2) is bounded as

$$
\left\|P(\alpha)\left(H_{A}(\alpha)-z\right) V\left(H_{A}(\alpha)-z\right)^{-1} Q(\alpha)\right\| \leqq C_{0}\|V\|_{\infty} B^{-1},
$$

$\forall \alpha \in S$. As for the second term, the commutator is (see (4.18))

$$
\left[V, H_{A}(\alpha)\right]=2 i(p-\alpha \nabla \rho-A) \cdot \nabla V-\Delta V .
$$

The resulting term in (6.2) involving $\Delta V$ is treated as above. As for the derivative term, it suffices to show

$$
\left\|\left(H_{A}(\alpha)-z\right)^{-1}\left(p_{i}-\alpha \partial_{i} \rho-A_{i}\right)\right\| \leqq C_{1} B^{1 / 2},
$$

for all $\alpha \in S$. To see this, let $V_{i}(\alpha) \equiv\left(p_{i}-\alpha \partial_{i} \rho-A_{i}\right)$ and $R(\alpha) \equiv\left(H_{A}(\alpha)-z\right)^{-1}$. For $u=R(\alpha) v, v \in L^{2}\left(\mathbb{R}^{2}\right)$, we have

$$
\begin{aligned}
\sum_{i=1}^{2}\left\|V_{i}(\alpha) u\right\|^{2} & =\left\langle u,\left\{H_{A}(\alpha)+2 i(\operatorname{Im} \alpha) \nabla \rho \cdot V(\alpha)\right\} u\right\rangle \\
& =\langle R(\alpha) v, v\rangle+z\|u\|^{2}+2 i(\operatorname{Im} \alpha)\langle u, \nabla \rho \cdot V(\alpha) u\rangle .
\end{aligned}
$$


This leads to a quadratic inequality for each $i=1,2$,

$$
\begin{aligned}
\left\|V_{i}(\alpha) R(\alpha) v\right\|^{2} \leqq & \|R(\alpha) v\|(\|v\|+|z|\|R(\alpha) v\|) \\
& +2|\operatorname{Im} \alpha|\|\nabla \rho\|_{\infty}\|R(\alpha) v\|\left\{\max _{j=1,2}\left\|V_{j}(\alpha) R(\alpha) v\right\|\right\} .
\end{aligned}
$$

Solving this, and noting that $|\operatorname{Im} \alpha| \leqq B^{1 / 2},|z|=\mathcal{O}(B)$, and, for this $z,\|R(\alpha)\|<C_{0}$ by (4.16)-(4.17), we get

$$
\left\|V_{i}(\alpha) R(\alpha)\right\| \leqq C_{1} B^{1 / 2}
$$

which is (6.4).

Lemma A.2. Let $\rho$ be the distance function defined in Sect. 4. $\exists$ constant $C>0$ depending only on $\left\|\partial^{\alpha} \rho\right\|_{\infty},|\alpha|=0,1,2$, such that $\forall \alpha \in S$,

$$
\left\|P(\alpha)^{\star} Q(\alpha)\right\| \leqq C B^{-1 / 2}|\operatorname{Im} \alpha|
$$

for $|\operatorname{Im} \alpha| \leqq B^{1 / 2}$.

Proof. We can assume that $\alpha$ is purely imaginary by a standard unitary equivalence argument (note that when $\alpha$ is real, $\left\|P(\alpha)^{\star} Q(\alpha)\right\|=0$.) Let $z \equiv B-1$, as in Lemma A.1. We then have by (6.3),

$$
\begin{aligned}
P(\alpha)^{\star} Q(\alpha)= & P(\alpha)^{\star}\left(H_{A}^{\star}(\alpha)-z\right)^{-1} Q(\alpha) \\
= & P(\alpha)^{\star}\left\{\left(H_{A}^{\star}(\alpha)-z\right)^{-1}-\left(H_{A}(\alpha)-z\right)^{-1}\right\} Q(\alpha) \\
& +P(\alpha)^{\star}\left(H_{A}(\alpha)-z\right)^{-1} Q(\alpha) .
\end{aligned}
$$

The last term is $\mathcal{O}\left(B^{-1}\right)$ by $(4.16)-(4.17)$. Let us write $\alpha \equiv i \beta$, with $\beta$ real. Then by the resolvent equation, we have

$$
\begin{aligned}
& \left(H_{A}^{\star}(\alpha)-z\right)^{-1}-\left(H_{A}(\alpha)-z\right)^{-1} \\
& \quad=-2 i \beta\left(H_{A}^{\star}(\alpha)-z\right)^{-1}[2(p-A) \cdot \nabla \rho+i \Delta \rho]\left(H_{A}(\alpha)-z\right)^{-1} .
\end{aligned}
$$

Using (6.8) in (6.7), we obtain the bound,

$$
\begin{aligned}
\left\|P(\alpha)^{\star} Q(\alpha)\right\| \leqq & 2|\operatorname{Im}(\alpha)|\left(\left\|\{2(p-A) \cdot \nabla \rho+i \Delta \rho\} P^{\star}(\alpha)\right\|+C_{0}\right) \\
& \times\left\|\left(H_{A}(\alpha)-z\right)^{-1} Q(\alpha)\right\| \\
\leqq & C_{1}|\operatorname{Im}(\alpha)| B^{-1}\left(\left\|2(p-A) \cdot \nabla \rho P^{\star}(\alpha)\right\|+C_{2}\right),
\end{aligned}
$$

where the constants are bounded as in the lemma. We again used (4.16)-(4.17) and the boundedness of $\Delta \rho$. The proof will follow from (6.9) once we show that

$$
\left\||p-A| P^{\star}(\alpha)\right\| \leqq C_{1} B^{1 / 2} .
$$

Inequality (6.10) follows directly as in (6.4) if we write

$$
\left(H_{A}^{\star}(\alpha)-z\right)^{-1}\left(p_{i}-A_{i}\right)=\left(H_{A}^{\star}(\alpha)-z\right)^{-1}\left\{\left(p_{i}+i \beta \partial_{i} \rho-A_{i}\right)-i \beta \partial_{i} \rho\right\},
$$

and note that $\beta \leqq B^{1 / 2}$. This proves (6.10). 


\section{Appendix 2}

In this appendix, we prove that there is almost surely spectrum near the band edges for the Hamiltonians $H_{\omega}$, defined in Sect. 2. This verifies that Theorem 2.1 is not trivial. To our knowledge, the existence of spectrum near the band edges does not follow from any known result. We prove the following theorem for the families $H_{\omega}$ as defined in Sect. 2. As in the previous sections, we restrict our discussion to the first Landau band although a similar calculation works for any band.

Theorem 7.1. Let $H_{\omega}$ be the family of Hamiltonians in Theorem 2.1 and let $\lambda_{ \pm}(B) \equiv B \pm M_{0}$ be the upper and lower first Landau band edges, respectively. Then for all $B$ sufficiently large, there exists a constant $C_{0}>0$, such that with probability one,

$$
\sigma\left(H_{\omega}\right) \cap\left[E-C_{0} B^{-1 / 2}, E+C_{0} B^{-1 / 2}\right] \neq \emptyset,
$$

for all $E \in\left[\lambda_{-}(B), \lambda_{+}(B)\right]$.

\section{Remarks 7.2.}

1. As the proof below shows, Theorem 7.1 remains valid if the assumptions (V1)-(V2) on the single-site potential $u$ are weakened. In particular, we only require that $u \in C_{0}^{2}\left(\mathbb{R}^{2}\right)$. The compactness assumption on supp $u$ can be weakened also provided $u$ has sufficiently fast decay. The proof can also be modified if the distribution function $g$ has non-compact support.

2. One can easily extend Theorem 7.1 to show that

$$
\sigma\left(H_{\omega}\right) \subset\left[\lambda_{-}(B)-C_{0} B^{-1 / 2}, \lambda_{+}(B)+C_{0} B^{-1 / 2}\right],
$$

with probability one.

3. Theorem 7.1 does not exclude the possibility that there are gaps of size $2 C_{0} B^{-1 / 2}$ in $\sigma\left(H_{\omega}\right)$.

Let $P_{0}$ be the Landau projection defined in (2.7) and set $Q_{0}=1-P_{0}$. We denote by $\phi_{0}$ the function in $\operatorname{Ran} P_{0}$ defined by

$$
\phi_{0}(x) \equiv\left(\frac{2}{\pi} B\right)^{1 / 2} e^{-B|x|^{2}}
$$

so that $P_{0} \phi_{0}=\phi_{0}$ and $\left\|\phi_{0}\right\|=1$.

Proof of Theorem 7.1. Let $E \in\left[\lambda_{-}(B), \lambda_{+}(B)\right]$ and choose any $\delta>0$. We will show that with non-zero probability there exists a state $\phi \in D\left(H_{A}\right)$, with $\|\phi\|=1$, and a constant $B_{0}>0$, such that for all $B>B_{0}$, there exists a uniform constant $C_{0}>0$ such that

$$
\left\|\left(H_{\omega}-E\right) \phi\right\| \leqq \delta+C_{0} B^{-1 / 2}
$$

This implies that

$$
\sigma\left(H_{\omega}\right) \cap\left[E-C_{0} B^{-1 / 2}-\delta, E+C_{0} B^{-1 / 2}+\delta\right] \neq \emptyset,
$$

with non-zero probability. Since $\sigma\left(H_{\omega}\right)$ is non-random and $\delta$ is arbitrary, the theorem follows.

Without loss of generality, we assume that $E \in\left[B, \lambda_{+}(B)\right]$. Let $r_{u}>0$ be the radius of the smallest ball centered at the origin containing $\operatorname{supp}(u)$ and let 
$\Lambda_{u} \equiv\left\{j \in \mathbb{Z}^{2},|j| \leqq 2 r_{u}\right\}$. Under condition (V1), we have $r_{u} \leqq 1 / \sqrt{2}$, but here we only need that $0<r_{u}<\infty$. We can write $M_{0}=\left\|V_{0}\right\|_{\infty}$, where $V_{0}(x)$ is defined by

$$
V_{0}(x) \equiv M \sum_{j \in \mathbb{Z}^{2},|j| \leqq 2 r_{u}} u_{j}(x)
$$

Since the potential $u$ is continuous and $\operatorname{supp} u$ is compact, there exists a point $x(E) \in \operatorname{supp} u$ such that $E-B=V_{0}(x(E))$. Using the magnetic translations (2.5), we can replace $u$ by another function $\tilde{u} \equiv u(\cdot-x(E))$, so that $x(E)=0$ and $V_{0}(0)=E-B$. Note that $|x(E)| \leqq 2 r_{u}$ for all energies in $\left[\lambda_{-}(B), \lambda_{+}(B)\right]$, and that $\|\tilde{u}\|_{\infty}=\|u\|_{\infty}$. Consequently, we will write $u$ for $\tilde{u}$ below and all constants will be uniform in $E$.

We use the function $\phi_{0}$ defined in (7.1) and write

$$
\left\|\left(H_{\omega}-E\right) \phi_{0}\right\| \leqq\left\|P_{0}\left[V_{\omega}-(E-B)\right] \phi_{0}\right\|+\left\|Q_{0} V_{\omega} \phi_{0}\right\| .
$$

By Lemma A.1, (6.1), there exists a constant $C_{1}$, depending only on $\left\|\partial^{\alpha} u\right\|_{\infty}$, $|\alpha|=0,1,2$, such that

$$
\left\|Q_{0} V_{\omega} \phi_{0}\right\| \leqq C_{1} B^{-1 / 2}
$$

To estimate the first term on the right side of (7.3), we write

$$
\begin{aligned}
\left\|P_{0}\left[V_{\omega}-(E-B)\right] \phi_{0}\right\| \leqq & \left\|\left[V_{\omega}-(E-B)\right] \phi_{0}\right\| \\
\leqq & \left\|\left[\sum_{j \in \mathbb{Z}^{2},|j| \leqq 2 r_{u}} \lambda_{j} u_{j}-(E-B)\right] \phi_{0}\right\| \\
& +M \sum_{j \in \mathbb{Z}^{2},|j| \geqq 2 r_{u}}\left\|u_{j} \phi_{0}\right\| .
\end{aligned}
$$

The last sum in (7.5) is estimated as follows. We first note that

$$
\begin{aligned}
\int_{\mathbb{R}^{2}} \phi_{0}(x)^{2} u^{2}(x-j) d^{2} x & \leqq \int_{\mathbb{R}^{2}}\left(\frac{2 B}{\pi}\right) e^{-2 B|x|^{2}} u^{2}(x-j) d^{2} x \\
& \leqq\left(\frac{2 B}{\pi}\right) \int_{\mathbb{R}^{2}} e^{-2 B|x+j|^{2}} u^{2}(x) d^{2} x \\
& \leqq\left(\frac{2 B}{\pi}\right)\|u\|_{\infty}^{2} e^{-2 B|j|^{2}+4 B r_{u}|j|} \int_{\mathbb{R}^{2}} e^{-2 B|x|^{2}} d^{2} x \\
& \leqq\|u\|_{\infty}^{2} e^{-2 B|j|^{2}+4 B r_{u}|j|} .
\end{aligned}
$$

It is easy to show that the sum over $\left\{j \in \mathbb{Z}^{2},|j| \geqq 2 r_{u}\right\}$ of the exponential on the last line in (7.6) is $\mathcal{O}\left(B^{-1 / 2}\right)$ for $B$ sufficiently large. It then follows from (7.6) that

$$
\sum_{j \in \mathbb{Z}^{2},|j| \geqq 2 r_{u}} M\left\|u_{j} \phi_{0}\right\| \leqq C_{2} B^{-1 / 2}
$$

for a constant $C_{2}$ uniform for all $B>B_{0}>0$ and $E \in\left[\lambda_{-}(B), \lambda_{+}(B)\right]$. 
It remains to estimate the first term on the right of the last line of (7.5). This is bounded above as

$$
\begin{aligned}
\left\|\left[\sum_{j \in \mathbb{Z}^{2},|j| \leqq 2 r_{u}} \lambda_{j} u_{j}-(E-B)\right] \phi_{0}\right\| \leqq & \left\|\sum_{j \in \mathbb{Z}^{2},|j| \leqq 2 r_{u}}\left(\lambda_{j}-M\right) u_{j} \phi_{0}\right\| \\
& +\left\|\left[V_{0}-(E-B)\right] \phi_{0}\right\| .
\end{aligned}
$$

For $\delta>0$ as given above, it follows from condition (V2) that

$$
\mathbb{P}\left\{\sum_{j \in \mathbb{Z}^{3},|j| \leqq 2 r_{u}}\left|\lambda_{j}-M\right| \leqq \delta\|u\|_{\infty}^{-1}\right\} \geqq\left[\int_{\varepsilon}^{M} g(\lambda) d \lambda\right]^{\left|\Lambda_{u}\right|},
$$

for $\varepsilon \equiv M-\delta\left(\|u\|_{\infty}\left|\Lambda_{u}\right|\right)^{-1}$. Consequently, for the first sum on the right we find the bound

$$
\left\|\sum_{j \in \mathbb{Z}^{2},|j| \leqq 2 r_{u}}\left(\lambda_{j}-M\right) u_{j} \phi_{0}\right\| \leqq \delta,
$$

with a non-zero probability. Finally, using the fact that $V_{0}(0)=E-B$, we find

$$
\begin{aligned}
\left\|\left[V_{0}-(E-B)\right] \phi_{0}\right\|^{2} & =\left(\frac{2 B}{\pi}\right) \int_{\mathbb{R}^{2}}\left|V_{0}(x)-(E-B)\right|^{2} e^{-2 B\|x\|^{2}} d^{2} x \\
& =\left(\frac{2}{\pi}\right) \int_{\mathbb{R}^{2}}\left|V_{0}\left(B^{-1 / 2} x\right)-(E-B)\right|^{2} e^{-2\|x\|^{2}} d^{2} x .
\end{aligned}
$$

Making use of the fact that $u \in C_{0}^{2}\left(\mathbb{R}^{2}\right)$ and that $V_{0}$ has compact support, the first order Taylor expansion for $V_{0}$ about $x=0$ gives

$$
\left|V_{0}\left(B^{-1 / 2} x\right)-V_{0}(0)\right| \leqq B^{-1 / 2}\|x\|\left\|\nabla V_{0}\right\|_{\infty} \leqq B^{-1 / 2} C_{3}
$$

where the constant $C_{3}$ depends only on $\left\|\partial^{\alpha} u\right\|_{\infty}$, for $\alpha=0,1$. Combining (7.7) $-(7.13)$, we find that

$$
\left\|P_{0}\left[V_{\omega}-(E-B)\right] \phi_{0}\right\| \leqq\left(C_{2}+C_{3}\right) B^{-1 / 2}+\delta,
$$

with positive probability. Returning to (7.3), this shows that

$$
\left\|\left(H_{\omega}-E\right) \phi_{0}\right\| \leqq \delta+C_{0} B^{-1 / 2},
$$

where $C_{0} \equiv C_{1}+C_{2}+C_{3}$, for all $B$ sufficiently large, and with positive probability.

Acknowledgements. We thank T. Hoffmann-Ostenhof and W. Thirring for their hospitality at the Erwin Schrödinger Institute in Vienna where most of this work was done. We thank R. Seiler and V. Jaksic for helpful discussions on the quantum Hall effect and A. Klein for many discussions on random operators. 


\section{References}

1. Halperin, B.I.: Quantized Hall conductance, current-carrying edge states, and the existence of extended states in a two-dimensional disordered potential. Phys. Rev. B 25, 2185 (1982)

2. Joynt, R., Prange, R.E.: Conditions for the quantum Hall effect. Phys. Rev. B 29, 3303-3317 (1984)

3. Kunz, H.: The quantum Hall effect for electrons in a random potential. Commun. Math. Phys. 112, 121-145 (1987)

4. Bellissard, J.: Ordinary quantum Hall effect and non-commutative cohomology. In: Localization in Disordered Systems. Weller, W., Zieche, P. (eds.) Leipzig: Teubner, 1988

5. Thouless, D.J.: Localization and the two-dimensional Hall effect. J. Phys. C 14, 3475-3480 (1981)

6. Brézin, E., Gross, D.J., Itzykson, C.: Nucl. Phys. B 235, 24 (1984)

7. Prange, R.E., Girvin, S.M. (eds.): The Quantum Hall Effect. Graduate Texts in Contemporary Physics. Berlin, Heidelberg, New York: Springer 1987

8. Combes, J.M., Hislop, P.D.: Localization for some continuous, random Hamiltonians in $d$-dimensions. J. Funct. Anal. 124, 149-180 (1994)

9. Howland, J.: Perturbation theory of dense point spectra. J. Funct. Anal. 74, 52-80 (1987)

10. Simon, B., Wolff, T.: Singular continuous spectrum under rank one perturbation and localization for random Hamiltonians. Comm. Pure Appl. Math. 39, 75-90 (1986)

11. von Dreifus, H., Klein, A.: A new proof of localization in the Anderson tight binding model. Commun. Math. Phys. 124, 285-299 (1989)

12. Spencer, T.: Localization for random and quasi-periodic potentials. J. Stat. Phys. 51, 1009$1019(1988)$

13. Wegner, F.: Z. Physik B 51, 279 (1983)

14. Laughlin, R.B.: Phys. Rev. B 23, 5632 (1981)

15. Carmona, R., Lacroix, J.: Spectral theory of random Schrödinger operators. Boston: Birkhäuser, 1990

16. Wang, W.M.: Développement asymptotique de la densité d'états pour l'opérateur de Schrödinger aléatoire avec champ magnetique. Séminaire des Equations aux Dérivées Partielles 1992-1993, Ecole Polytechnique, Exposé XVIII (1993)

17. Wang, W.M.: Private communication, July 1994 and Microlocalization, Percolation and Anderson localization for the Magnetic Schrödinger operator with a random potential, to appear in J. Funct. Anal.

18. Kotani, S., Simon B.: Localization in general one-dimensional systems. II. Commun. Math. Phys. 112, 103-120 (1987)

19. Combes, J.M., Hislop, P.D., Mourre, E.: Spectral Averaging, Perturbation of Singular Spectrum, and Localization. Preprint 1994, to appear in Trans. Amer. Math. Soc.

20. Combes, J.M., Hislop, P.D.: Localization for Long-Range and Correlated Potentials. In preparation

21. Grimmett, G.: Percolation. Berlin, Heidelberg, New York: Springer, 1989

22. Chayes J.L., Chayes L.: Percolation and Random Media. In: Critical Phenomena, Random Systems, and Gauge Theories, Proc. les Houches Summer School, Session XLIII, 1000-1142 (1984)

23. Kato, T.: Perturbation theory for linear operators. 2nd ed., Berlin, Heidelberg, Berlin: Springer, 1980

24. Pulé J.: Communication at the IAMP satellite conference on Disordered Systems, Paris, July 1994 and Dorlas, T.C., Macris, N., Pulé, J.V.: Localisation in a single-band approximation to random Schrödinger operators in a Magnetic field, to appear in Helv. Phys. Acta. 
